\title{
Some families of $q$-vector fields on path spaces
}

\author{
K. D. Elworthy and Xue-Mei Li
}

\begin{abstract}
Some families of $H$-valued vector fields with calculable Lie brackets are given. These provide examples of vector fields on path spaces with a divergence and we show that versions of Bismut type formulae for forms on a compact Riemannian manifold arise as projections of the infinite dimensional theory.
\end{abstract}

Let $M$ be a compact Riemannian manifold and $\left\{P_{t}: t \geq 0\right\}$ its heat semigroup acting on differential forms. Thus if $\phi$ is a bounded measurable (or square integrable) form on $M$ we have

$$
\begin{cases}\frac{\partial}{\partial t}\left(P_{t} \phi\right) & =\frac{1}{2} \Delta\left(P_{t} \phi\right), \quad t>0 \\ P_{0} \phi & =\phi\end{cases}
$$

where we use the sign convention

$$
\Delta=-(d \delta+\delta d) .
$$

The formula for the exterior derivative of $P_{t} \phi$

$$
d\left(P_{t} \phi\right)\left(V_{0}\right)=\frac{1}{t} \mathbb{E} \phi\left(W_{t}^{(q)} \int_{0}^{t}\left(W_{s}^{(q)}\right)^{-1} \iota_{\left\langle-, d x_{s}\right\rangle} W_{s}^{(q+1)}\left(V_{0}\right)\right)
$$

where $\phi$ is a bounded measurable $q$-form and $V_{0} \in \wedge^{q+1} T_{x_{0}} M$ was given in [EL98], extending Bismut's formula for the special case of $q=0$, [Bis84]

$$
d\left(P_{t} f\right)\left(V_{0}\right)=\frac{1}{t} \mathbb{E} f\left(x_{t}\right) \int_{0}^{t}\left\langle W_{s}^{(1)}\left(V_{0}\right), d x_{s}\right\rangle
$$

key words: path space, differential forms, Riemannian manifolds, heat equation, integration by parts, Bismut formula, Malliavin calculus.

Research partially supported by NSF research grant DMS 0072387, EPSRC GR/NOO 845, the Alexander von Humboldt stiftung. 
for $f: M \rightarrow \mathbb{R}$ bounded and measurable and $V_{0} \in T_{x_{0}} M$. In these formulae the expectation $\mathbb{E}$ is with respect to a Brownian motion $\left\{x_{t}: 0 \leq t<\infty\right\}$ on $M$ starting from $x_{0}$ and for $q=1,2, \ldots$ we use the 'damped' parallel translations $W_{s}^{(q)}: \wedge^{q} T_{x_{0}} M \rightarrow \wedge^{q} T_{x_{s}} M, 0 \leq s<\infty$ of $q$-vectors along the sample paths of $(x$.) given by the covariant equation along $(x$.):

$$
\left\{\begin{aligned}
\frac{D}{\partial s}\left(W_{s}^{(q)}\left(V_{0}\right)\right) & =-\frac{1}{2} \mathcal{R}_{x_{0}}^{q}\left(W_{s}^{(q)}\left(V_{0}\right)\right) \\
W_{0}^{(q)}\left(V_{0}\right) & =V_{0}
\end{aligned}\right.
$$

where $\mathcal{R}_{x}^{q}: \wedge^{q} T_{x} M \rightarrow \wedge^{q} T_{x} M$ is the q-th Weitzenbock curvature defined by

$$
\Delta \phi=\nabla^{*} \nabla \phi-\phi\left(\mathcal{R}^{q}-\right)
$$

for $\phi$ a smooth $q$-form. See, for example, [Air76], [Elw82], [IW81] and [Elw88]. In particular $\mathcal{R}^{1}$ is the Ricci curvature $\operatorname{Ric}^{\#}: T M \rightarrow T M$, i.e. $\left\langle\mathcal{R}_{x}^{1} v, u\right\rangle=$ $\operatorname{Ric}(v, u), u, v \in T_{x} M$.

Formula (1) has been refined and extended by Driver and Thalmaier [DT01], giving analogous results on various operators on vector bundles over $M$, e.g. the square of the Dirac operator. Rather different types of Bismut type formulae for covariant derivatives of operators on vector bundles were obtained by Norris in [Nor93] by different methods. The original proof of (1) in [EL98] was to derive it by applying the method of conditional expectation from [EY93] to the earlier, non-intrinsic, formula of Li, [Li92], [EL94], see also [Elw92],

$$
d\left(P_{t} \phi\right)_{x_{0}}=\frac{1}{t} \mathbb{E} \int_{0}^{t}\left\langle T \xi_{s}-, d x_{s}\right\rangle \wedge \xi_{t}^{*}(\phi)
$$

for $\phi$ a bounded measurable $q$-form. Here $\left\{\xi_{s}: 0 \leq s<\infty\right\}$ is a gradient Brownian flow on $M$ (see below), $x_{t}=\xi_{t}\left(x_{0}\right)$ for $x_{0} \in M, T \xi_{t}: T M \rightarrow T M$ is the (random) derivative of $\xi_{t}$, and $\xi_{t}^{*} \phi$ is the pull back of $\phi$ :

$$
\begin{aligned}
\xi_{t}^{*} \phi(V): & =\phi\left(\wedge^{q}\left(T \xi_{t}\right)(V)\right) \\
& =\phi\left(T \xi_{t}\left(v^{1}\right) \wedge \cdots \wedge T \xi_{t}\left(v^{q}\right)\right), \quad V=v^{1} \wedge \cdots \wedge v^{q} \in \wedge^{q} T M
\end{aligned}
$$

In fact the same proof allows the use of any Brownian flow which has Levi-Civita connection as its LeJan-Watanabe connection in the sense of [ELL99]. All the formulae can be extended to obtain differentiation formulae for the corresponding heat kernels and this is done in the references cited. 
Bismut's formula (2) can be obtained from infinite dimensional integration by parts formulae by considering the cylindrical functions $F(\sigma)=.f\left(\sigma_{t}\right)$ on the space of paths over $M$ and indeed Driver's integration by parts formula can be derived from it, as described in [EL96] following comments by Nualart. Since integration by parts theorems for forms on path spaces are not yet well understood it is interesting to ask if analogous results hold for (1) and (4) in the context of the $L^{2}$ theory of differential forms being developed in [EL00] and [EL] and to derive (1) and (4) by the methods used there. Our approach is very much in the spirit of Bismut's approach to Malliavin Calculus [Bis81] and of Fang-Franchi for Lie groups [FF97]. We do not attack the two challenges of : (i) deriving integration by parts formulae for forms on path spaces from (1) and (4), as done for functions in [EL96]; and (ii) deriving the more general results of Driver and Thalmaier by similar methods.

In $\S 1$ below we define the class of flows used in (1). In $\S 2$ the infinite dimensional theory in [EL00], [EL] is briefly described, and in $\S 3$ the two parts are combined to both give a proof of (1) and (2) and to give some interesting examples of q-vector fields on path spaces which have explicitly calculable divergences. On the way we obtain, from our stochastic differential equation (5), a family of $\mathrm{H}$ vector fields on Wiener space, which for gradient stochastic differential equations form a commuting family and in certain other cases have easily computable Lie brackets. This gives a $q+1$-vector field $\tilde{V}_{0}$ on the path space $C_{x_{0}} M$ of $M$ which has a divergence with respect to the Wiener measure $\mu_{x_{0}}$ in the sense that the divergence $\operatorname{div} \tilde{V}_{0}$ is a vector field on $C_{x_{0}} M$ satisfying equation (15) below and such that (1) can be written:

$$
\int_{C_{x_{0}} M} d \phi^{t}\left(\tilde{V}_{0}\right) d \mu_{x_{0}}=-\int_{C_{x_{0}} M} \phi^{t}\left(\operatorname{div} \tilde{V}_{0}\right) d \mu_{x_{0}}
$$

for $\phi^{t}$ the cylindrical $q$-form on $C_{x_{0}} M$ obtained from a differential form $\phi$ on the manifold $M$ :

$$
\phi^{t}(V)=\phi\left(x_{t}\right)\left(V_{t, \ldots, t}\right), \quad \text { for } V \text { any } q \text {-vector field. }
$$

Various versions of formula (2) are derived from this. For invariant stochastic differential equations on Lie groups no conditioning is needed in (4), c.f. [FF97], and we obtain (46). Using gradient systems we give in (41) the minor extension to (4) mentioned in [EL98] to the case of semigroups on forms with generators of the form $\frac{1}{2} \Delta+\mathcal{L}_{A}$. We also consider the connections used by Ikeda and Watanabe in [IW81], sometimes known as Riemann-Cartan-Weyl connections [RS84], where 
the formulae (2) and (4) have a pleasant form (47), although the infinitesimal generators of the semigroup on forms are rather complicated. However the main aims of the article are to study some interesting classes of $q$-vector fields on $C_{x_{0}} M$ and to show that some finite dimensional formulae can be considered as projections of the infinite dimensional theory.

\section{Stochastic flows and LeJan-Watanabe connections}

Consider a Stratonovich stochastic differential equation

$$
d x_{t}=X\left(x_{t}\right) \circ d B_{t}+A\left(x_{t}\right) d t
$$

on $M$ driven by a $m$-dimensional Brownian motion $\left\{B_{t}, 0 \leq t<\infty\right\}$. Here $X: M \times \mathbb{R}^{m} \rightarrow T M$ and $A: M \rightarrow T M$ are assumed to be smooth and $X(x):=X(x,-): \mathbb{R}^{m} \rightarrow T_{x} M$ is assumed to be linear, surjective for each $x$ and to induce the inner product $\langle,\rangle_{x}$ on $T_{x} M$ given by the Riemannian structure of $M$. This implies the Markov process of solutions to (5) has infinitesimal generator of the form

$$
\mathcal{A}=\frac{1}{2} \Delta+\mathcal{L}_{Z}
$$

where $\mathcal{L}_{Z}$ is Lie differentiation in the direction of some vector field $Z$ :

$$
\mathcal{L}_{Z}(f)(x)=d f(Z(x)), \quad x \in M .
$$

The solution to (5) from $x$ shall be denoted by $\left\{\xi_{t}(x), 0 \leq t<\infty\right\}$. Write $x_{t}=\xi_{t}\left(x_{0}\right)$. We can, and will, take versions which makes $\xi_{t}(x)$ continuous in $t$ and a $C^{\infty}$ diffeomorphism of $M$ in $x$.

Let $Y: T M \rightarrow \mathbb{R}^{m}$ be the adjoint of $X$, i.e. $Y_{x}=X(x)^{*}$, so that $Y$ is the right inverse to $X$. As described in [ELJL97], or more generally in [ELL99], $X$ induces a metric connection $\breve{\nabla}$ on $M$ defined by

$$
\breve{\nabla}_{v} U=X(x) d\left[x \mapsto Y_{x}(U(x))\right](v), \quad v \in T_{x} M
$$

for any smooth vector field $U$ on $M$. In general this, which we call the LeJanWatanabe connection of our stochastic differential equation, has torsion. The torsion is given by

$$
\breve{T}(u, v)=X(x) d Y(u, v), \quad u \cdot v \in T_{x} M
$$


where $d Y$ refers to the exterior derivative of $Y$ considered as an $\mathbb{R}^{m}$-valued differential 1-form on $M$, see Proposition 2.2.3 in [ELL99]. When the torsion vanishes $\breve{\nabla}$ is just the Levi-Civita connection, which we will always refer to using $\nabla, \frac{D}{\partial t}$ etc. There are two principal classes of examples, Example 1.1 and Example 1.2 below, for which this holds.

\section{Example 1.1. Gradient system.}

Here we consider an isometric immersion $\alpha: M \rightarrow \mathbb{R}^{m}$ for some $m$, e.g. by using Nash's theorem. Let $X(x): \mathbb{R}^{m} \rightarrow T_{x} M$ be the orthogonal projection identifying $T_{x} M$ with its image in $\mathbb{R}^{m}$ under $d \alpha$. Then $Y_{x}: T_{x} M \rightarrow \mathbb{R}^{m}$ is just the inclusion $(d \alpha)_{x}$ and (7) is the classical formula for the Levi-Civita connection of a sub-manifold of $\mathbb{R}^{m}$.

\section{Example 1.2. Riemannian symmetric spaces.}

Let $M$ be a symmetric space $(K, H, \sigma)$. In particular $K$ is a Lie group acting transitively on $M$ and $H$ can be identified with the isotropy subgroup fixing the point $x_{0}$ of $M$, so that $k \mapsto k x_{0}$ gives a diffeomorphism of $K / H$ with $M$. Assume that the Lie algebra $\mathfrak{k}$ of $K$ has an inner product $\langle,\rangle_{\mathfrak{k}}$, say, invariant under the adjoint action of $K$ on $\mathfrak{k}$. The action induces a stochastic differential equation

$$
X: M \times \mathfrak{k} \rightarrow T M
$$

with $X(x) e=\frac{d}{d t}[(\exp (t e)) \cdot x]_{t=0}$. It is surjective and so induces a Riemannian structure $\left(\langle,\rangle_{x}, x \in M\right)$ on $M$ which is $K$-invariant. The LeJan-Watanabe connection $\breve{\nabla}$ is the Levi-Civita connection for the Riemannian structure, as is proved in Corollary 1.4.9 of [ELL99].

An important class of examples which give connections other than the LeviCivita connections are the left invariant, and the right invariant stochastic differential equations on Lie groups. For $M$ a Lie group with left invariant metric take the Lie algebra $\mathfrak{g}$ on $\mathbb{R}^{m}$ and define

$$
X^{L}: G \times \mathfrak{g} \rightarrow T G
$$

to be left invariant with $X^{L}(e) \alpha=\alpha$, for $e$ the identity element of $G$, i.e.

$$
X^{L}(g) \alpha=T L_{g}(\alpha)
$$


for $L_{g}: G \rightarrow G$ the left translation $x \mapsto g \cdot x$. Similarly if $R_{g}$ denotes right translation, $x \mapsto x \cdot g$, define $X^{R}: G \times \mathfrak{g} \rightarrow T G$ by $X^{R}(g) \alpha=T R_{g}(\alpha)$. It is easy to see that the associated connections in the sense of (7) are just the canonical left and right invariant connections, $\nabla^{L}$ and $\nabla^{R}$ say, of $G$, respectively. The flow $\xi_{t}^{R}$ for the left invariant stochastic differential equation $d x_{t}=X^{L}\left(x_{t}\right) \circ d B_{t}$, where $B_{t}$ is a $\mathfrak{g}$-valued Brownian motion, is given by

$$
\xi_{t}^{L}(x)=x g_{t}, \quad x \in G, t \geq 0 .
$$

Here $g_{t}$ is the solution starting from the identity $e$ to $d g_{t}=X^{L}\left(g_{t}\right) \circ d B_{t}$. Similarly the solution $\xi_{t}^{R}(x)$, with $\xi_{0}^{R}(x)=x$ to the right invariant stochastic differential equation $d x_{t}=X^{R}\left(x_{t}\right) \circ d B_{t}$ is given by the right translation of $h_{t}$, the solution starting from $e$. If the metric on $G$ is bi-invariant both stochastic differential equations, with $A \equiv 0$, have Brownian motions as solutions. Also in that case the connections are torsion skew symmetric, with $\nabla^{L}$ and $\nabla^{R}$ adjoint to each other. In particular the torsions $T^{L}$ and $T^{R}$ are given by

$$
T^{L}(u, v) \equiv-[U, V](g), \quad T^{R}(u, v)=[U, V](g),
$$

where $U, V$ are left invariant vector fields with $u=U(g)$ and $v=V(g)$.

In the bi-invariant case we can also treat $G$ as a symmetric space by taking $K=G \times G$ with action $\left(g_{1}, g_{2}\right) x=g_{1} x g_{2}^{-1}$, with $H=\Delta G \equiv\{(g, g) \in K$ : $g \in G\}$ the isotropy subgroup fixing the identity $e$ of $G$. The symmetry map $\sigma: \mathfrak{g} \times \mathfrak{g} \rightarrow \mathfrak{g}$ is given by $\sigma(\alpha, \beta)=(\beta, \alpha)$. The stochastic differential equation induced by the action as described in Example 1.2 above is just

$$
d x_{t}=T R_{x_{t}} \circ d B_{t}-T L_{x_{t}} \circ d B_{t}^{\prime}
$$

where $\left(B_{t}\right)$ and $\left(B_{t}^{\prime}\right)$ are two independent Brownian motions on $\mathfrak{g}$. The solution flow is then given by $\xi_{t}(x)=g_{t} x\left(g_{t}^{\prime}\right)^{-1}$ where $g_{t}$ and $g_{t}^{\prime}$ are independent Brownian motions on $G$ and solutions to the following right invariant stochastic differential equations respectively:

$$
\begin{array}{ll}
d g_{t}=T R_{g_{t}} \circ d B_{t}, & g_{0}=e, \\
d g_{t}^{\prime}=T R_{g_{t}^{\prime}} \circ d B_{t}^{\prime}, & g_{0}=e,
\end{array}
$$

The solutions $\xi_{t}(x)$, when $A \equiv 0$, are also Brownian motions on $G$.

For more general homogeneous spaces $M=K / H$, if the metric on $M$ is induced from an $\operatorname{ad}_{K}$-invariant inner product on $\mathfrak{k}$ and the orthogonal complement of 
the ker $X\left(x_{0}\right)$ is $\operatorname{ad}_{H}$ invariant then $\breve{\nabla}$, the connection associated to the stochastic differential equation defined as in Example 1.2 above, is $K$-invariant. (see [ELL99], Proposition 1.2.9). Again in that case

$$
\xi_{t}(x)=k_{t} x, \quad x \in M
$$

for $k_{t}$ the solution to the equation $d k_{t}=T R_{k_{t}} \circ d B_{t}$ on $K$ with initial point $k_{0}=e$.

Stochastic flows and differential forms. For $\left\{\xi_{t}: t \geq 0\right\}$ the flow of our stochastic differential equation (5) there are semigroups on differential forms defined by $P_{t} \phi=\mathbb{E} \xi_{t}^{*} \phi$. On $q$-forms the semigroup has infinitesimal generator $\mathcal{A}^{q}$ given on smooth forms by

$$
\mathcal{A}^{q} \phi=\frac{1}{2} \sum_{j=1}^{m} \mathcal{L}_{X^{j}} \mathcal{L}_{X^{j}} \phi+\mathcal{L}_{A}(\phi)
$$

for $\mathcal{L}_{X^{j}}$ denoting the Lie differentiation in the direction $X^{j}$, etc and with $X^{j}$ denoting the vector fields $X(\cdot) e^{j}$ for $e^{1}, \ldots e^{m}$ the standard basis of $\mathbb{R}^{m}$. This comes from Itô's formula, see [Elw92] or [Elw88]. The generator can be written

$$
\mathcal{A}^{q}(\phi)=-(d \hat{\delta}+\hat{\delta} d) \phi+\mathcal{L}_{A}(\phi)
$$

where, for $V \in \wedge^{q-1} T M$,

$$
\begin{aligned}
\hat{\delta} \phi(V) & =-\sum_{j=1}^{m}\left(\iota_{X^{j}} \mathcal{L}_{X^{j}} \phi\right)(V) \\
& =-\operatorname{trace} \hat{\nabla}_{-} \phi(-, V),
\end{aligned}
$$

and $\hat{\nabla}$ is the adjoint connection to $\breve{\nabla}$, in the sense of Driver [Dri92] so the torsion of $\hat{\nabla}$ is $-\breve{T}$. See [ELL99]. This shows $\mathcal{A}^{q}$ depends only on the connection $\breve{\nabla}$ and the Riemannian metric on $T M$. In particular if $A \equiv 0$ in (5) and the associated connection $\breve{\nabla}$ is the Levi-Civita connection we get the usual Hodge-Kodaira Laplacian, c.f. [Kus88].

\section{Spaces of forms on the path space of $M$}

Fix $T>0$ and $x_{0} \in M$ and let $C_{x_{0}} M$ denote the Banach manifold of continuous paths $\sigma:[0, T] \rightarrow M$ with $\sigma(0)=x_{0}$. Let $\mu_{x_{0}}$ be the measure induced on it by the solution to (5) starting from $x_{0}$. 
We can identify the tangent space $T_{\sigma} C_{x_{0}} M$ to $C_{x_{0}} M$ at $\sigma$ with the space of continuous $v:[0, T] \rightarrow T M$ with $v(0)=0$ such that $v(t) \in T_{\sigma(t)} M$ for each $0 \leq t \leq T$. For $q=1,2, \ldots$, let $\wedge^{q} T_{\sigma} C_{x_{0}} M$ be the space of anti-symmetric $q$-vectors at $x_{0}$ completed using the largest reasonable cross norm, so that its dual space can be identified with the Banach space of continuous alternating $q$-linear maps $\phi: T_{\sigma} C_{x_{0}} M \times \cdots \times T_{\sigma} C_{x_{0}} M \rightarrow \mathbb{R}$. Thus $q$-forms on $C_{x_{0}} M$ are sections of the dual bundle $\left(\wedge^{q} T_{\sigma} C_{x_{0}} M\right)^{*}$, e.g. see [Lan62].

As an example of a $q$-vector on $C_{x_{0}} M$ consider $\wedge^{q}\left(T_{x_{0}} \xi\right)(U)$ evaluated on a fixed $\omega \in \Omega$, where $U \in \wedge^{q} T_{x_{0}} M$. It is then a $q$-vector at $\left\{x_{t}(\omega): 0 \leq t \leq T\right\}$. For $q=1$ it is the tangent vector $\left\{T_{x_{0}} \xi_{t}(\cdot, \omega)(U): 0 \leq t \leq T\right\}$ to $C_{x_{0}} M$ at $x .(\omega)$. For higher $q$ we identify $q$-vectors $V$ with certain continuous maps $V:[0, T]^{q} \rightarrow$ $\otimes{ }^{q} T M$ such that $V_{t_{1}, t_{2}, \ldots, t_{q}} \in T_{\sigma\left(t_{1}\right)} \otimes \cdots \otimes T_{\sigma\left(t_{q}\right)} M$. Then, generalizing to allow $U$ to be in $\wedge^{q} C\left([0, T] ; T_{x_{0}} M\right)$,

$$
\wedge^{q+1}\left(T_{x_{0}} \xi\right)(U)_{t_{1}, \ldots, t_{q+1}}=\left(T_{x_{0}} \xi_{t_{1}} \otimes \cdots \otimes T_{x_{0}} \xi_{t_{q+1}}\right)\left(U_{t_{1}, \ldots, t_{q}}\right)
$$

As $\omega$ varies in $\Omega$ such $q+1$ vectors do not strictly speaking form $q+1$ vector fields on $C_{x_{0}} M$. However we can obtain $q$-vector fields from them by conditioning, 'filtering out the redundant noise', to give $\sigma \mapsto \mathbb{E}\left\{\wedge^{q+1}\left(T_{x_{0}} \xi\right)(U) \mid \xi .\left(x_{0}, \omega\right)=\sigma\right\}$. As shorthand, we write:

$$
\overline{\wedge^{q+1}\left(T_{x_{0}} \xi\right)_{\sigma}}(U)=\mathbb{E}\left\{\wedge^{q+1}\left(T_{x_{0}} \xi\right)(U) \mid \xi .\left(x_{0}, \omega\right)=\sigma\right\} .
$$

See [EY93],[ELL99]. Below we shall often use $\bar{f}$ denote the conditional expectation of a random function $f$ with respect to the filtration of $\left\{x_{t}: 0 \leq t<\infty\right\}$.

An important class of forms on $C_{x_{0}} M$ are the cylindrical forms. In particular if $\phi$ is a $q$-form on $M$ and $t \in[0, T]$ we have the cylindrical $q$-form $\phi^{t}$ on $C_{x_{0}} M$ given by $\phi^{t}(V)=\phi\left(V_{t, \ldots, t}\right)$.

For $\psi$ a $(q-1)$-form on $M$ we see, with $P_{t}$ as in the last section,

$$
\left.P_{t}(d \psi)=\mathbb{E}(d \psi)\left(\wedge^{q}\left(T_{x} \xi_{t}\right) U\right)=\int_{C_{x_{0}} M}(d \psi)^{t}\left(\overline{\wedge^{q} T_{x} \xi}\right)(U)\right) d \mu_{x_{0}}(\sigma)
$$

In general we say that an $L^{2} q$-vector field $V$ has a divergence if there is an $L^{2}$ $(q-1)$-vector field $\operatorname{div} V$ on $C_{x_{0}} M$ such that for all smooth cylindrical $(q-1)$ forms $\phi$ on $C_{x_{0}} M$ we have

$$
\int_{C_{x_{0}} M} d \phi(V) d \mu_{x_{0}}=-\int_{C_{x_{0}} M} \phi(\operatorname{div} V) d \mu_{x_{0}} .
$$


For $U=u^{1} \wedge \cdots \wedge u^{q}$, a primitive vector, the non-intrinsic Bismut type formula (4) will follow from showing that $\overline{\wedge^{q}\left(T_{x_{0}} \xi\right)}(U)$ has a divergence with

$$
\begin{aligned}
& \operatorname{div}\left(\overline{\wedge^{q} T_{x} \xi_{\sigma}}(U)\right)_{t, \ldots, t} \\
& =\frac{1}{t} \mathbb{E}\left\{\sum_{j=1}^{q}(-1)^{j}\left(\int_{0}^{t}\left\langle T \xi_{s}\left(u^{j}\right), d x_{s}\right\rangle\right) \wedge^{q-1}\left(T \xi_{t}\right)\left(u^{1} \wedge \ldots \widehat{u^{j}} \cdots \wedge u^{q}\right)\right. \\
& \quad \mid \xi .(x .)=\sigma\}
\end{aligned}
$$

at least for a gradient stochastic differential equation with no drift. Here we have adopted the convention that $\widehat{u^{j}}$ means the omission of the vector $u^{j}$ in the tensor. For a general $q$-vector $U$ in $\wedge^{q} T_{x_{0}} M$ this could be written

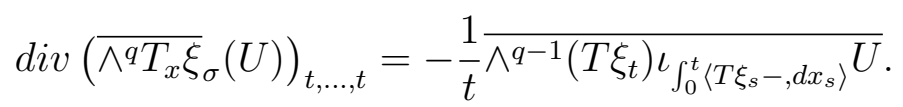

Equation (1) will follow, as in [EL98], by calculating the conditional expectations. Note that we have used another convention: if $\ell$ is a linear map on a vector space

$$
\iota_{\ell}\left(b^{1} \wedge \cdots \wedge b^{q}\right)=\sum_{j=1}^{q}(-1)^{j+1} \ell\left(b^{j}\right) b^{1} \wedge \cdots \wedge \widehat{b^{j}} \wedge \cdots \wedge b^{q} .
$$

In [EL], see also [EL00], a general theory for forms on path spaces is given. There we define Hilbert spaces $\mathcal{H}^{(q)}$, densely included in $\wedge^{q} T_{\sigma} C_{x_{0}} M$ and show that suitably regular sections of $\mathcal{H}^{(q)}$ do have a divergence and give evidence to suggest that the divergence is a section of $\mathcal{H}^{(q-1)}$, with proofs for $q=2,3$. This is the major step in the construction of an $L^{2}$ deRham complex on $C_{x_{0}} M$, with an $L^{2}$ Hodge decomposition and analogue of the Hodge-Kodaira Laplacian. For this construction we take a stochastic differential equation (5) on $M$ whose solutions are Brownian motions and with its associated connection $\breve{\nabla}$ the Levi-Civita connection, for example by taking an isometric embedding in $\mathbb{R}^{m}$ and $A \equiv 0$. We take $\left(B\right.$.) to be the canonical Brownian motion defined up to time $T$ so that $C_{0} \mathbb{R}^{m}$ with Wiener measure $\mathbb{P}$ is the underlying probability space. For fixed $x_{0} \in M$ there is the Itô map

$$
\begin{aligned}
\mathcal{I}: C_{0} \mathbb{R}^{m} & \rightarrow C_{x_{0}} M \\
\mathcal{I}(\omega)_{t} & =\xi_{t}\left(x_{0}, \omega\right) .
\end{aligned}
$$

It is smooth in the sense of Malliavin Calculus with $H$-derivative

$$
T_{\omega} \mathcal{I}: H \rightarrow T C_{x_{0}} M
$$


defined for almost all $\omega \in C_{0} \mathbb{R}^{m}$. Here $H$ is the Cameron-Martin space; $H=$ $L_{0}^{2,1}\left([0, T] ; \mathbb{R}^{m}\right)$. We have the formula of Bismut

$$
T \mathcal{I}(h)_{t}=T \xi_{t} \int_{0}^{t}\left(T \xi_{s}\right)^{-1} X\left(x_{s}\right) \dot{h}_{s} d s
$$

By an $L^{2} H$-q-vector field on $C_{0} \mathbb{R}^{m}$ we mean an element $U \in L^{2}\left(C_{0} \mathbb{R}^{m} ; \wedge^{q} H\right)$ where $\wedge^{q} H$ refers to the completed tensor product using the usual Hilbert space cross norm. Given such a vector field $U$ we can define

$$
\left(\wedge^{q} T \mathcal{I}\right)(U): C_{0} \mathbb{R}^{m} \rightarrow \wedge^{q} T C_{x_{0}} M
$$

At $\omega \in C_{0} \mathbb{R}^{m}$ this is in $\wedge^{q} T_{x .(\omega)} C_{x_{0}} M$.

To obtain a $q$-vector field on $C_{x_{0}} M$ define, for $\sigma \in C_{x_{0}} M$

$$
{\overline{\left(\wedge^{q} T \mathcal{I}\right)(U)_{\sigma}}}_{\sigma}=\mathbb{E}\left\{\left(\wedge^{q} T \mathcal{I}\right)(U) \mid x .=\sigma\right\}
$$

Taking constant $U$, that is $U(\omega) \equiv h$, some $h \in H$ we obtain continuous linear maps

$$
{\overline{\left(\wedge^{q} T \mathcal{I}\right)_{\sigma}}}: \wedge^{q} H \rightarrow \wedge^{q} T_{\sigma} C_{x_{0}} M
$$

defined by

$$
{\overline{\left(\wedge^{q} T \mathcal{I}\right)_{\sigma}}}(h)={\overline{\left(\wedge^{q} T \mathcal{I}\right)(h)_{\sigma}}} .
$$

We can then define $\mathcal{H}_{\sigma}^{q}=\operatorname{Image}\left({\overline{\wedge^{q} T \mathcal{I}_{\sigma}}}\right)$ with its induced Hilbert space structure, inner product $\langle,\rangle_{\sigma}$, so that $\left(\overline{\wedge^{q} T \mathcal{I}}\right)_{\sigma}$ becomes an isometry. These spaces are defined for almost all $\sigma \in C_{x_{0}} M$. It is shown in [EL] that they depend only on the Riemannian structure of $M$. The fact that suitably regular $L^{2}$ sections of $\mathcal{H}^{q}$ have a divergence can be deduced from the theory for Wiener spaces given by Shigekawa [Shi86].

The following is a specialization of his basic result to primitive adapted $q$ vector fields.

Theorem 2.1 (Shigekawa [Shi86]) For $q>1$, let $\phi: C_{0} \mathbb{R}^{m} \rightarrow\left(\wedge^{q} H\right)^{*}$ be an $H$-differential q-form on Wiener space and $h^{i}: C_{0} \mathbb{R}^{m} \rightarrow H, i=1$ to q adapted vector fields such that $h:=h^{1}(\cdot) \wedge \cdots \wedge h^{q+1}(\cdot)$ is in $\mathbb{D}^{1,2}\left(\wedge^{q+1} H\right)$. If also $\phi \in \mathbb{D}^{1,2}\left(\left(\wedge^{q} H\right)^{*}\right)$ then

$$
\int_{C_{0} \mathbb{R}^{m}} d \phi(h) d \mathbb{P}=-\int_{C_{0} \mathbb{R}^{m}} \phi(\text { divh }) d \mathbb{P}
$$


where

$$
\begin{aligned}
\operatorname{divh}= & \sum_{j=1}^{q}(-1)^{j} \int_{0}^{T}\left\langle\dot{h}_{s}^{j}, d B_{s}\right\rangle\left(h^{1} \wedge \cdots \wedge \widehat{h^{j}} \wedge \cdots \wedge h^{q}\right) \\
& -\sum_{1 \leq i<j \leq q+1}(-1)^{i+j}\left[h^{i}, h^{j}\right] \wedge h^{1} \wedge \cdots \wedge \widehat{h}^{i} \wedge \cdots \wedge \widehat{h^{j}} \wedge \cdots \wedge h^{q+1} .
\end{aligned}
$$

The assumptions of primitivity of $h$ or adaptness of the components could have been omitted with Skorohod integrals replacing Itô integrals, see [Shi86].

The extension to forms on $C_{x_{0}} M$ in [EL00], [EL] and the proof we give below for formulae (1) and (2) is based on the following observation:

Theorem 2.2 Let $\psi$ be a smooth cylindrical q-form on $C_{x_{0}} M$. Then for $h \in$ $\mathbb{D}^{1,2}\left(\wedge^{q+1} H\right)$ of the form $h=h^{1} \wedge \cdots \wedge h^{q+1}$ with each $h^{i}$ adapted, the q-vector field $\overline{\wedge^{q+1} T \mathcal{I}(h)}$ on $C_{x_{0}} M$ has a divergence:

$$
\int_{C_{x_{0}} M} d \psi\left(\overline{\wedge^{q+1} T \mathcal{I}(h)}\right) d \mu_{x_{0}}=-\int_{C_{x_{0}} M} \psi\left(\operatorname{div} \overline{\wedge^{q} T \mathcal{I}(h)}\right) d \mu_{x_{0}}
$$

and

$$
\operatorname{div}\left(\overline{\wedge^{q+1} T \mathcal{I}(h)}\right)=\overline{\wedge^{q T \mathcal{I}}(\operatorname{divh})} .
$$

Proof. The pull back form $\mathcal{I}_{*}(\psi)$ is in $\mathbb{D}^{1,2}\left(\left(\wedge^{q} H\right)^{*}\right)$. The integration by parts formula of Shigekawa, Theorem 2.1, applies to give:

$$
\begin{gathered}
\int_{C_{x_{0}} M} d \psi\left(\overline{\wedge^{q+1} T \mathcal{I}(h)}\right) d \mu_{x_{0}}=\int_{C_{0} \mathbb{R}^{m}} d\left(\mathcal{I}_{*} \psi\right)(h) d \mathbb{P} \\
=-\int_{C_{0} \mathbb{R}^{m}} \mathcal{I}_{*} \psi(\text { divh }) d \mathbb{P}=-\int_{C_{x_{0}} M} \psi\left(\overline{\wedge^{q} T \mathcal{I}(\text { divh })}\right) d \mu_{x_{0} .}
\end{gathered}
$$

For other discussions of differential forms on path spaces see [JL91], [Kus92] and [Lea] together with the references there. 


\section{Some families of $H$-valued vector fields}

3.1 The Lie brackets. Below we identify a family of vector fields whose Lie brackets behave particularly well.

Proposition 3.1 Given tangent vectors $b_{i} \in T_{x_{0}} M, i=1,2$ and $\rho:[0, T] \rightarrow \mathbb{R}, a$ non-random $L^{1}$ function, set:

$$
h^{i}=\int_{0}^{\cdot} \rho(r) Y_{x_{r}}\left(T \xi_{r}\left(b^{i}\right)\right) d r .
$$

Then the Lie bracket of the two vector fields $h^{i}$ on the Wiener space $C_{0}\left(\mathbb{R}^{m}\right)$ is given by:

$$
\begin{aligned}
\frac{d}{d t}\left[h^{1}, h^{2}\right]_{t} & =\rho(t)\left(\int_{0}^{t} \rho(r) d r\right)(d Y)\left(T \xi_{t}\left(b^{1}\right), T \xi_{t}\left(b^{2}\right)\right) \\
& +\rho(t) Y_{x_{t}}\left(T \xi_{t} \int_{0}^{t} \rho(r)\left(T \xi_{r}\right)^{-1} X\left(x_{r}\right) d Y\left(T \xi_{r}\left(b^{1}\right), T \xi_{r}\left(b^{2}\right)\right) d r\right) .
\end{aligned}
$$

and so

$T \mathcal{I}_{t}\left(\left[h^{1}, h^{2}\right]\right)=\left(\int_{0}^{t} \rho(s) d s\right) T \xi_{t}\left(\int_{0}^{t} \rho(s) T \xi_{s}^{-1} X\left(x_{s}\right) d Y\left(T \xi_{s}\left(b^{1}\right), T \xi_{s}\left(b^{2}\right)\right) d s\right)$.

Proof. Denote by $D$ differentiation on Euclidean spaces, $D^{H}$ the H-derivatives of Wiener functionals, $\nabla$ covariant differentiation using the Levi-Civita connection on $M$, and $\nabla^{H}$ the corresponding covariant H-derivatives. Below we regularly abuse notation by omitting $\omega$ in the argument, especially with $\xi_{t}$ and $T \xi_{t}$. First observe that

$$
\begin{aligned}
T \mathcal{I}_{t}\left(h^{i}\right)(\omega) & =T \xi_{t} \int_{0}^{t}\left(T \xi_{r}\right)^{-1} X\left(x_{r}(\omega)\right)\left(\dot{h}_{r}^{i}\right) d r \\
& =\left(\int_{0}^{t} \rho(r) d r\right) T \xi_{t}\left(b^{i}\right)
\end{aligned}
$$

and that

$$
\begin{aligned}
\frac{d}{d t}\left[h^{1}, h^{2}\right]_{t}(\omega) & =\frac{d}{d t}\left(D^{H} h_{t}^{2}(\omega)\left(h^{1}(\omega)\right)-D^{H} h_{t}^{1}(\omega)\left(h^{2}(\omega)\right)\right) \\
& =D^{H} \dot{h}_{t}^{2}(\omega)\left(h^{1}(\omega)\right)-D^{H} \dot{h}_{t}^{1}(\omega)\left(h^{2}(\omega)\right) .
\end{aligned}
$$


Now

$$
\begin{aligned}
& D^{H} \dot{h}_{t}^{2}(\omega)\left(h^{1}(\omega)\right) \\
= & D^{H}\left(Y_{x_{t}}\left(T \xi_{t}\left(\rho(t) b^{2}\right)\right)\right)(\omega)\left(h^{1}(\omega)\right) \\
= & \rho(t)\left(\nabla_{h^{1}(\omega)}^{H} Y_{x_{t}}\right)\left(T \xi_{t}\left(b^{2}\right)\right)+\rho(t) Y_{x_{t}(\omega)}\left(\nabla_{h^{1}(\omega)}^{H}\left(T \xi_{t}\left(b^{2}\right)\right)\right) \\
= & \rho(t)\left(\nabla_{T_{\omega} \mathcal{I}_{t}\left(h^{1}\right)} Y\right)\left(T \xi_{t}\left(b^{2}\right)\right)+\rho(t) Y_{x_{t}(\omega)}\left(\nabla_{h^{1}(\omega)}^{H}\left(T \xi_{t}\left(b^{2}\right)\right)\right) .
\end{aligned}
$$

By the expression (22) for $T \mathcal{I}_{t}\left(h^{1}\right)$, we have

$$
\begin{aligned}
D^{H} \dot{h}_{t}^{2}(\omega)\left(h^{1}(\omega)\right)= & \rho(t)\left(\int_{0}^{t} \rho(r) d r\right) \nabla_{T \xi_{t}\left(b^{1}\right)} Y\left(T \xi_{t}\left(b^{2}\right)\right) \\
& +\rho(t) Y_{x_{t}(\omega)}\left(\nabla_{h^{1}(\omega)}^{H}\left(T \xi_{t}\left(b^{2}\right)\right)\right) .
\end{aligned}
$$

To calculate the second term, consider the map

$$
\begin{array}{cl}
\tilde{\mathcal{I}}_{t}: & M \times C_{0}\left(R^{m}\right) \rightarrow M \\
\tilde{\mathcal{I}}_{t}(x, \omega) & =\xi_{t}(x)(\omega)
\end{array}
$$

with $T_{(x, \omega)} \tilde{\mathcal{I}}_{t}: T_{x} M \times L_{0}^{2,1}\left(\mathbb{R}^{m}\right) \rightarrow T_{\xi_{t}(x)(\omega)} M$ its derivative in the sense of Malliavin calculus. Then

$$
T_{(x, \omega)} \tilde{\mathcal{I}}_{t}(u, h)=T_{x} \xi_{t} \int_{0}^{t}\left(T_{x} \xi_{r}\right)^{-1} X\left(\xi_{r}(x)\right)\left(\dot{h}_{r}\right) d r+T \xi_{t}(u),
$$

and $T_{\left(x_{0}, \omega\right)} \tilde{\mathcal{I}}_{t}(u, h(\omega))=T \mathcal{I}_{t}(h(\omega))+T \xi(u)$. Since $b^{2}$ is non-random,

$$
\begin{aligned}
& Y_{x_{t}(\omega)}\left(\nabla_{h^{1}(\omega)}^{H}\left(T \xi_{t}\left(b^{2}\right)\right)\right)=Y_{x_{t}(\omega)}\left(\nabla_{h^{1}(\omega)}^{H}\left(T \xi_{t}\right)\right)\left(b^{2}\right) \\
= & Y_{\left.x_{t}(\omega)\right)} \nabla_{b^{2}}\left[T_{(-, \omega)} \tilde{\mathcal{I}}\left(0, h^{1}(\omega)\right)\right]_{t}
\end{aligned}
$$

But

$$
\begin{aligned}
& \nabla_{b^{2}}\left[T_{(-, \omega)} \tilde{\mathcal{I}}\left(0, h^{1}(\omega)\right)\right]_{t} \\
= & \nabla_{b^{2}}\left[T_{-} \xi_{t} \int_{0}^{t}\left(T_{-} \xi_{r}\right)^{-1} X\left(\xi_{r}(-, \omega)\right)\left(\dot{h}_{r}^{1}(\omega)\right) d r\right] \\
= & \nabla_{b^{2}}\left[T_{-} \xi_{t} \int_{0}^{t}\left(T_{-} \xi_{r}\right)^{-1} X\left(\xi_{r}(-)\right) \rho(r) Y_{x_{r}}\left(T \xi_{r}\left(b^{1}\right)\right) d r\right] .
\end{aligned}
$$


Since $\left(T_{-} \xi_{r}\right)^{-1} X\left(\xi_{r}(-, \omega)\right) Y_{\xi_{r}(-)}\left(T_{-} \xi_{r}\right)=I d$,

$$
\begin{aligned}
& \nabla_{b^{2}}\left[T_{(-, \omega)} \tilde{\mathcal{I}}\left(0, h^{1}(\omega)\right)\right]_{t} \\
= & \left(\int_{0}^{t} \rho(r) d r\right) \nabla_{b^{2}}\left(T \xi_{t}\right)\left(b^{1}\right) \\
& -T_{x_{0}} \xi_{t} \int_{0}^{t}\left(T_{x_{0}} \xi_{r}\right)^{-1} X\left(x_{r}\right) \nabla_{b^{2}}\left(\rho(r) Y_{\xi_{r}(-)}\left(T_{-} \xi_{r}\left(b^{1}\right)\right)\right) \\
= & \left(\int_{0}^{t} \rho(r) d r\right) \nabla_{b^{2}}\left(T \xi_{t}\right)\left(b^{1}\right)-T_{x_{0}} \xi_{t} \int_{0}^{t} \rho(r)\left(T_{x_{0}} \xi_{r}\right)^{-1} \nabla_{b^{2}}\left(T_{-} \xi_{r}\right)\left(b^{1}\right) \\
& -T_{x_{0}} \xi_{t} \int_{0}^{t} \rho(r)\left(T_{x_{0}} \xi_{r}\right)^{-1} X\left(x_{r}\right) \nabla_{b^{2}}\left(Y_{\xi_{r}(-)}\right)\left(T_{x_{0}} \xi_{r}\left(b^{1}\right)\right) \\
= & \left(\int_{0}^{t} \rho(r) d r\right) \nabla_{b^{2}}\left(T \xi_{t}\right)\left(b^{1}\right)-T \xi_{t} \int_{0}^{t} \rho(r)\left(T \xi_{r}\right)^{-1} \nabla_{b^{2}}\left(T \xi_{r}\right)\left(b^{1}\right) d r \\
& -T \xi_{t} \int_{0}^{t} \rho(r)\left(T \xi_{r}\right)^{-1} X\left(x_{r}\right)\left(\nabla_{T \xi_{r}\left(b^{2}\right)} Y\right)\left(T \xi_{r}\left(b^{1}\right)\right) d r
\end{aligned}
$$

and so

$$
\begin{aligned}
& \nabla_{b^{2}}\left[T_{(-, \omega)} \tilde{\mathcal{I}}\left(0, h^{1}(\omega)\right)\right]_{t}-\nabla_{b^{1}}\left[T_{(-, \omega)} \tilde{\mathcal{I}}\left(0, h^{2}(\omega)\right)\right]_{t} \\
= & \left(\int_{0}^{t} \rho(r) d r\right)\left(\nabla_{b^{2}}\left(T \xi_{t}\right)\left(b^{1}\right)-\nabla_{b^{1}}\left(T \xi_{t}\right)\left(b^{2}\right)\right) \\
& -T \xi_{t} \int_{0}^{t} \rho(r)\left(T \xi_{r}\right)^{-1}\left(\nabla_{b^{2}}\left(T \xi_{r}\right)\left(b^{1}\right)-\nabla_{b^{1}}\left(T \xi_{r}\right)\left(b^{2}\right)\right) d r \\
& -T \xi_{t} \int_{0}^{t} \rho(r)\left(T \xi_{r}\right)^{-1} X\left(x_{r}\right) d Y\left(T \xi_{r}\left(b^{2}\right), T \xi_{r}\left(b^{1}\right)\right) d r \\
= & -T \xi_{t} \int_{0}^{t} \rho(r)\left(T \xi_{r}\right)^{-1} X\left(x_{r}\right) d Y\left(T \xi_{r}\left(b^{2}\right), T \xi_{r}\left(b^{1}\right)\right) d r .
\end{aligned}
$$

Finally combining (23), (24) and the last identity, we have 


$$
\begin{aligned}
& \frac{d}{d t}\left[h^{1}, h^{2}\right]_{t}(\omega) \\
= & \rho(t)\left(\int_{0}^{t} \rho(r) d r\right)\left(\nabla_{T \xi_{t}\left(b^{1}\right)} Y\right)\left(T \xi_{t}\left(b^{2}\right)\right) \\
& -\rho(t)\left(\int_{0}^{t} \rho(r) d r\right)\left(\nabla_{T \xi_{t}\left(b^{2}\right)} Y\right)\left(T \xi_{t}\left(b^{1}\right)\right) \\
& -\rho(t) Y_{x_{t}}\left(T \xi_{t} \int_{0}^{t} \rho(r)\left(T \xi_{r}\right)^{-1} X\left(x_{r}\right) d Y\left(T \xi_{r}\left(b^{2}\right), T \xi_{r}\left(b^{1}\right)\right) d r\right) \\
= & \rho(t)\left(\int_{0}^{t} \rho(r) d r\right) d Y\left(T \xi_{t}\left(b^{1}\right), T \xi_{t}\left(b^{2}\right)\right) \\
& +\rho(t) Y_{x_{t}}\left(T \xi_{t} \int_{0}^{t} \rho(r)\left(T \xi_{r}\right)^{-1} X\left(x_{r}\right) d Y\left(T \xi_{r}\left(b^{1}\right), T \xi_{r}\left(b^{2}\right)\right) d r\right) .
\end{aligned}
$$

To prove (21), simply do an integration by parts:

$$
\begin{aligned}
T \mathcal{I}_{t}\left(\left[h^{1}, h^{2}\right]\right)= & T \xi_{t} \int_{0}^{t} \rho(s)\left(\int_{0}^{s} \rho(r) d r\right)\left(T \xi_{s}\right)^{-1} X d Y\left(T \xi_{s}\left(b^{1}\right), T \xi_{s}\left(b^{2}\right)\right) d s \\
& +T \xi_{t} \int_{0}^{t} \rho(s) \int_{0}^{s} \rho(r)\left(T \xi_{r}\right)^{-1} X d Y\left(T \xi_{r}\left(b^{1}\right), T \xi_{r}\left(b^{2}\right)\right) d r d s \\
= & T \xi_{t} \int_{0}^{t} \rho(s)\left(\int_{0}^{s} \rho(r) d r\right)\left(T \xi_{s}\right)^{-1} X d Y\left(T \xi_{s}\left(b^{1}\right), T \xi_{s}\left(b^{2}\right)\right) d s \\
& +T \xi_{t}\left(\int_{0}^{t} \rho(s) d s\right) \int_{0}^{t} \rho(r)\left(T \xi_{r}\right)^{-1} X d Y\left(T \xi_{r}\left(b^{1}\right), T \xi_{r}\left(b^{2}\right)\right) d r d s \\
& -T \xi_{t} \int_{0}^{t}\left(\int_{0}^{s} \rho(r) d r\right) \rho(s)\left(T \xi_{s}\right)^{-1} X d Y\left(T \xi_{s}\left(b^{1}\right), T \xi_{s}\left(b^{2}\right)\right) d s \\
= & \left(\int_{0}^{t} \rho(s) d s\right) T \xi_{t}\left(\int_{0}^{t} \rho(s)\left(T \xi_{s}\right)^{-1} X d Y\left(T \xi_{s}\left(b^{1}\right), T \xi_{s}\left(b^{2}\right)\right) d s\right) .
\end{aligned}
$$

Note that if $\breve{T}$ is the torsion of the connection $\breve{\nabla}$, then $X(d Y)=\breve{T}$ by (8). Consequently, 
Corollary 3.2 For $h^{i}$ defined by (8) in Proposition 3.1,

$$
\begin{aligned}
X\left(x_{t}\right) \frac{d}{d t}\left[h^{1}, h^{2}\right]_{t}= & \rho(t)\left(\int_{0}^{t} \rho(r) d r\right) \breve{T}\left(T \xi_{t}\left(b^{1}\right), T \xi_{t}\left(b^{2}\right)\right) \\
& +\rho(t) T \xi_{t} \int_{0}^{t} \rho(r)\left(T \xi_{r}\right)^{-1} \breve{T}\left(T \xi_{r}\left(b^{1}\right), T \xi_{r}\left(b^{2}\right)\right) d r
\end{aligned}
$$

and

$$
T \mathcal{I}_{t}\left(\left[h^{1}, h^{2}\right]\right)=\left(\int_{0}^{t} \rho(s) d s\right) T \xi_{t}\left(\int_{0}^{t} \rho(s) T \xi_{s}^{-1} \breve{T}\left(T \xi_{s}\left(b^{1}\right), T \xi_{s}\left(b^{2}\right)\right) d s\right) .
$$

We shall now return to our examples.

\section{Example 3.1. Gradient system.}

In Example 1.1, where the stochastic differential equation is a gradient system, we have $d Y \equiv 0$. Consequently $\frac{d}{d t}\left[h^{1}, h^{2}\right]_{t}(\omega)$ vanishes and so does $\left[h^{1}, h^{2}\right]_{t}(\omega)$. Indeed in this case if we set

$$
h^{v}=\int_{0}^{\cdot} Y_{x_{r}}\left(T \xi_{r}(v)\right) d r
$$

for $v \in T_{x_{0}} M$, we have a commuting family $\left\{h^{v}: v \in T_{x_{0}} M\right\}$ of $\mathcal{H}$-valued vector fields on $C_{0} \mathbb{R}^{m}$. Note that if $\alpha: M \rightarrow \mathbb{R}^{m}$ is the immersion defining our stochastic differential equation, then $\alpha \circ \xi_{t}$ is a random map of $M$ into $\mathbb{R}^{m}$ and $\dot{h}_{t}^{v}=d\left(\alpha \circ \xi_{t}\right)(v)=\left(\xi_{t}\right)_{*}(d \alpha)(v)$.

\section{Example 3.2. The Lie group case.}

For $M$ a Lie group with left invariant stochastic differential equations corresponding to a bi-invariant metric as discussed in $\S 1$, if $v \in \mathfrak{g}$ and $x_{0}=e$ the identity element, we have

$$
\begin{aligned}
h^{i} & :=\int_{0}^{\cdot} \rho(r) Y_{x_{r}}^{L}\left(T \xi_{r}^{L}\left(b^{i}\right)\right) d r \\
& =\int_{0}^{\cdot} \rho(r) T L_{x_{r}}^{-1} T R_{x_{r}}\left(b^{i}\right) d r \\
& =\int_{0}^{\cdot} \rho(r) a d\left(x_{r}^{-1}\right)\left(b^{i}\right) d r
\end{aligned}
$$


In this case

$$
T \mathcal{I}_{t}\left(h^{i}\right)=T R_{x_{t}} \int_{0}^{t} a d\left(x_{r}\right) \dot{h}_{r}^{i} d r=T R_{x_{t}}\left(\int_{0}^{t} \rho(r) d r b^{i}\right) .
$$

Let $T^{R}$ and $T^{L}$ be the torsions for the left and right invariant connections respectively. From above, (9), the fact that $T \xi_{t}^{L}(u)=T R_{x_{t}}(u)$, and by the right invariance of $T R$,

$$
\begin{aligned}
\frac{d}{d t}\left[h^{1}, h^{2}\right]_{t}= & -\rho(t)\left(\int_{0}^{t} \rho(r) d r\right) T L_{x_{t}}^{-1} T R_{x_{t}}\left[b^{1}, b^{2}\right] \\
& -\rho(t) T L_{x_{t}}^{-1} T R_{x_{t}} \int_{0}^{t} \rho(r)\left[b^{1}, b^{2}\right] d r \\
= & -2 \rho(t)\left(\int_{0}^{t} \rho(r) d r\right) a d\left(x_{t}^{-1}\right)\left[b^{1}, b^{2}\right] \\
= & -\frac{d}{d t}\left(\int_{0}^{t} \rho(r) d r\right)^{2} a d\left(x_{t}^{-1}\right)\left[b^{1}, b^{2}\right] \\
= & -a d\left(x_{t}^{-1}\right) \frac{d}{d t}\left[\int_{0}^{t} \rho(r) d r b^{1}, \int_{0}^{t} \rho(r) d r b^{2}\right]
\end{aligned}
$$

Note that

$$
\begin{aligned}
T \mathcal{I}_{t}\left(\left[h^{1}, h^{2}\right]\right) & =-T R_{x_{t}} \int_{0}^{t} a d\left(x_{r}\right)\left(\frac{d}{d r}\left[h^{1}, h^{2}\right]_{r}\right) d r \\
& =-T R_{x_{t}} \int_{0}^{t} 2 \rho(r)\left(\int_{0}^{r} \rho(s) d s\right) d r\left[b^{1}, b^{2}\right] \\
& =-T R_{x_{t}}\left[\int_{0}^{t} \rho(r) d r b^{1}, \int_{0}^{t} \rho(r) d r b^{2}\right] .
\end{aligned}
$$

\section{Example 3.3. Lie group as a symmetric space.}

We now consider the stochastic differential equation (10):

$$
d x_{t}=T R_{x_{t}} \circ d B_{t}-T L_{x_{t}} \circ d B_{t}^{\prime}
$$

on the Lie group $G$ with bi-invariant metric. The derivative flow of the solution $\xi_{t}$ is given by $T \xi_{t}=T R_{\left(g_{t}^{\prime}\right)^{-1}} T L_{g_{t}}$. Note that in our notation the map, giving the 
equation (10), $X(g): \mathfrak{g} \times \mathfrak{g} \rightarrow T_{x} M$ is given by $X(g)\left(\alpha_{1}, \alpha_{2}\right)=T R_{g}\left(\alpha_{1}\right)-$ $T L_{g}\left(\alpha_{2}\right)$ with inverse $Y: T G \rightarrow \mathfrak{g} \times \mathfrak{g}$ given, for $u \in T_{g} G$, by

$$
\begin{aligned}
Y(u) & =\left(T R_{g}^{-1}(u),-T L_{g}^{-1}(u)\right) \\
& =\left(Y^{R} u,-Y^{L} u\right) .
\end{aligned}
$$

Thus if $u, v \in T_{g} G$, for $T^{R}$ and $T^{L}$ the torsion tensors as in (9),

$$
\begin{aligned}
d Y(u, v) & =\left(T R_{g}^{-1} T^{R}(u, v), T L_{g}^{-1} T^{L}(u, v)\right) \\
& =\left(\left[Y^{R}(u), Y^{R}(v)\right],\left[Y^{L}(u), Y^{L}(v)\right]\right)
\end{aligned}
$$

so

$$
\begin{aligned}
\frac{d}{d t}\left[h^{1}, h^{2}\right]_{t}=\rho(t)\left(\int_{0}^{t} \rho(r) d r\right)( & {\left[Y^{R}\left(T L_{g_{t}} T R_{g_{t}^{\prime}}^{-1} b^{1}\right), Y^{R}\left(T L_{g_{t}} T R_{g_{t}^{\prime}}^{-1} b^{2}\right)\right], } \\
& {\left.\left[Y^{L}\left(T L_{g_{t}} T R_{g_{t}^{\prime}}^{-1} b^{2}\right), Y^{L}\left(T L_{g_{t}} T R_{g_{t}^{\prime}}^{-1} b^{1}\right]\right)\right) . }
\end{aligned}
$$

Now

$$
\begin{aligned}
Y^{R}\left(T L_{g_{t}} T R_{\left(g_{t}^{\prime}\right)}^{-1} b^{i}\right) & =\left(T R_{g_{t}}\left(g_{t}^{\prime}\right)^{-1}\right)^{-1} T L_{g_{t}} T R_{g_{t}^{\prime}}^{-1} b^{i} \\
& =\left(T R_{g_{t}^{\prime}}^{-1} \circ T R_{g_{t}}\right)^{-1} T L_{g_{t}} T R_{g_{t}^{\prime}}^{-1} b^{i} \\
& =a d\left(g_{t}\right) b^{i} .
\end{aligned}
$$

while

$$
\begin{aligned}
Y^{L}\left(T L_{g_{t}} T R_{\left(g_{t}^{\prime}\right)}^{-1} b^{i}\right) & =\left(T L_{g_{t}}\left(g_{t}^{\prime}\right)^{-1}\right)^{-1} T L_{g_{t}} T R_{g_{t}^{\prime}}^{-1} b^{i} \\
& =T L_{g_{t}^{\prime}} T R_{g_{t}^{\prime}}^{-1} b^{i}=a d\left(g_{t}^{\prime}\right) b^{i},
\end{aligned}
$$

so

$$
\begin{aligned}
\frac{d}{d t}\left[h^{1}, h^{2}\right]_{t} & =\rho(t)\left(\int_{0}^{t} \rho(r) d r\right)\left(\operatorname{ad}\left(g_{t}\right)\left[b^{1}, b^{2}\right], a d\left(g_{t}^{\prime}\right)\left[b^{1}, b^{2}\right]\right) \\
& \in \mathfrak{g} \times \mathfrak{g} .
\end{aligned}
$$

In this case $h_{t}^{i}=\left(\int_{0}^{t} \rho(r) a d\left(g_{r}\right) b^{i} d r,-\int_{0}^{t} \rho(r) a d\left(g_{r}^{\prime}\right) b^{i} d r\right)$.

3.2 A family of $\mathcal{H}^{2}$-valued vector fields. In general when the associated connection $\breve{\nabla}$ for (5) is the Levi-Civita connection $\nabla$ and $A \equiv 0$ we expect that 
$\overline{\wedge^{q} T \mathcal{I}\left(h^{1} \wedge \cdots \wedge h^{q}\right)_{\sigma}} \in \mathcal{H}_{\sigma}^{q}$ for almost all $\sigma \in C_{x_{0}} M$. It follows from [EL00] that this holds for $q=1$ and from [EL] for $q=2$, and a proof for $q=2$ for our special $h^{i}$ 's is given below in Proposition 3.3 as an illustrative example. For general $q$ we have,

$$
\overline{\wedge^{q}(T \mathcal{I})\left(h^{1} \wedge \cdots \wedge h^{q}\right)}=\mathbb{E}\left\{\wedge^{q}(T \xi .)\left(b^{\rho, 1} \wedge \cdots \wedge b^{\rho, q}\right) \mid x_{s}: 0 \leq s \leq T\right\}
$$

where $b_{t}^{\rho, j}=\left(\int_{0}^{t} \rho(r) d r\right) b^{j}$ and $(T \xi).\left(b^{\rho, j}\right)$ is just the vector field

$\left\{\left(T \xi_{t}\right)\left(b_{t}^{\rho, j}\right): 0 \leq t \leq T\right\}$ along $\xi .\left(x_{0}\right)$. From [ELL99] or [EY93] for gradient systems, we can deduce that for $0<t_{1}<\cdots<t_{q} \leq T$

$$
\begin{aligned}
& \overline{\wedge^{q} T \mathcal{I}\left(h^{1} \wedge \cdots \wedge h^{q}\right)_{t_{1}, \ldots, t_{q}}} \\
& =\int_{0}^{t_{1}} \rho(r) d r \int_{0}^{t_{2}} \rho(r) d r \ldots \int_{0}^{t_{q}} \rho(r) d r \cdot\left(\mathbf{1} \otimes \cdots \otimes W_{t_{, q}}^{t_{q-1}}\right) \\
& \left(\mathbf{1} \otimes \cdots \otimes W_{t_{q-1}}^{(2)^{t_{q-2}}}\right) \ldots W_{t_{1}}^{(q)}\left(b^{1} \wedge \cdots \wedge b^{q}\right) .
\end{aligned}
$$

Here $W^{(q)}{ }_{t}^{s}=W_{t}^{(q)} \circ\left(W_{s}^{(q)}\right)^{-1}: \wedge^{q} T_{x_{s}} M \rightarrow \wedge^{q} T_{x_{t}} M$ with $W_{t}^{s}=W^{(1)}{ }_{t}^{s}$, for $W^{(q)}$ the damped parallel transport defined by (3).

Let us recall the characterization of $\mathcal{H}^{1}$ and $\mathcal{H}^{2}$. For $q=2$ it is shown in [EL], that

$$
\mathcal{H}_{\sigma}^{2}=\left\{U+Q_{\sigma}(U) \in \wedge^{q} T_{\sigma} C_{x_{0}} M: U \in \wedge^{2} \mathcal{H}_{\sigma}^{1}\right\}
$$

where $Q_{\sigma}: \wedge^{2} \mathcal{H}_{\sigma}^{1} \rightarrow \wedge^{2} T_{\sigma} C_{x_{0}} M$ is defined by

$$
Q(U)_{s, t}=\left(\mathbf{1} \otimes W_{t}^{s}\right) W_{s}^{(2)} \int_{0}^{s}\left(W_{r}^{(2)}\right)^{-1} \mathcal{R}\left(U_{r, r}\right) d r, \quad 0 \leq s \leq t \leq T
$$

for $\mathcal{R}: \wedge^{2} T M \rightarrow \wedge^{2} T M$ the curvature operator.

The space $\mathcal{H}^{1}$ is the 'Bismut tangent space', [JL91], but with a different Hilbert space structure. In fact elements of $\mathcal{H}^{1}$ are exactly vector fields $v$ in $M$ along $\sigma$ such that $\|v\|_{\sigma}:=\int_{0}^{T}\left|\frac{\mathbb{D}}{d t} v_{t}\right|_{\sigma(t)}^{2} d t<\infty$ where

$$
\frac{\mathbb{D}}{d t} v_{t}:=\frac{D}{d t} v_{t}+\frac{1}{2} \operatorname{Ric}^{\#}\left(v_{t}\right)=W_{t}^{-1} \frac{d}{d t} W_{t}^{-1} V_{t} .
$$

From this it follows that $\wedge^{2} \mathcal{H}_{\sigma}^{1}$ consists of those $U$ in $\wedge^{2} T_{\sigma} C_{x_{0}} M$ for which

$$
\left(\wedge^{2}(W .)^{-1}(U)\right)_{s, t}=\int_{0}^{s} \int_{0}^{t} H\left(r_{1}, r_{2}\right) d r_{1} d r_{2}
$$

for some $H$ in $L^{2}\left([0, T] \times[0, T] ; T_{x_{0}} M \otimes T_{x_{0}} M\right)$. 
Proposition 3.3 Let $\lambda \in L_{0}^{2,1}([0, T] ; \mathbb{R})$. Take $V$ in $\wedge^{2} T_{x_{0}} M$ and $V^{\lambda} \in \wedge^{2} L_{0}^{2,1} T_{x_{0}} M$ defined by $V_{s, t}^{\lambda}=\lambda(s) \lambda(t) V$. If $A \equiv 0$ in (5) and the associated connection is the Levi-Civita connection, the vector field $Z$, where

$$
Z=\mathbb{E}\left\{\wedge^{2}(T \xi)\left(V^{\lambda}\right) \mid x_{s}, 0 \leq s \leq T\right\}
$$

forms a section of $\mathcal{H}^{2}$. It is equal to $U+Q(U)$ for $Q$ as above and $U$ given by

$$
\begin{aligned}
U_{s, t} & =\left(\mathbf{1} \otimes W_{t}^{s}\right) \lambda(s) \lambda(t) W_{s}^{(2)}(V) \\
& -\left(W_{s} \otimes W_{t}\right) \int_{0}^{s} \lambda^{2}(r) \wedge^{2}\left(W_{r}\right)^{-1} \mathcal{R}\left(W_{r}^{(2)}(V)\right) d r
\end{aligned}
$$

for all $0 \leq s \leq t \leq T$. It has divergence

$$
\begin{aligned}
(\operatorname{div} Z)_{t}= & -\int_{0}^{T}\left(\left\langle\frac{\mathbb{D}}{\partial r}-, d x_{r}\right\rangle \otimes \mathbf{1}\right) Z_{r, t} \\
& \left.+W_{t} \int_{0}^{t}\left(\left\langle-, d x_{r}\right\rangle \otimes\left(W_{r}\right)^{-1}\right) \mathcal{R}\left(\mathbf{1} \otimes W_{t}^{r}\right)\right)^{-1} Z_{r, t} .
\end{aligned}
$$

(For an interpretation of the, apparently adapted, integrals, see the proof below.)

Proof. From (27), for $0 \leq s \leq t \leq T$,

$$
Z_{s, t}=\lambda(s) \lambda(t)\left(\mathbf{1} \otimes W_{t}^{s}\right) W_{s}^{(2)}(V) .
$$

So

$$
\left[\left(\wedge^{2} W .\right)^{-1} Z\right]_{s, t}=\lambda(s) \lambda(t)\left(\wedge^{2} W_{s}\right)^{-1} W_{s}^{(2)}(V) .
$$

Setting

$$
k(r)=\wedge^{2}\left(W_{r}^{-1}\right) W_{r}^{(2)}(V) \in \wedge^{2} T_{x_{0}} M
$$

we see that

$$
\left[\left(\wedge^{2} W .\right)^{-1} Z\right]_{s, t}=\lambda(s) \lambda(t) k(s \wedge t), \quad \text { for all } s, t \in[0, T] .
$$

Set

$$
\tilde{U}=\wedge^{2}\left(W^{-1}\right)(U)
$$

We only need to show that

$$
\tilde{U}+\left(\wedge^{2} W^{-1}\right) Q(U)=\lambda(s) \lambda(t) k(s \wedge t)
$$


and that $U$ belongs to $\wedge^{2} \mathcal{H}^{1}$.

Now for any $K:[0, T] \times \Omega \rightarrow \wedge^{2} T M$ over $\left\{x_{t}: 0 \leq t \leq T\right\}$

$$
\wedge^{2}\left(W_{r}\right) \frac{d}{d r}\left(\wedge^{2}\left(W_{r}\right)^{-1} K_{r}\right)=\mathcal{R}\left(K_{r}\right)+W_{r}^{(2)} \frac{d}{d r}\left[\left(W_{r}^{(2)}\right)^{-1} K_{r}\right]
$$

when the derivative exists, so $k^{\prime}(s)=\wedge^{2}\left(W_{s}^{-1}\right) \mathcal{R}\left(W_{s}^{(2)}(V)\right)$ and consequently by (30)

$$
\tilde{U}_{s, t}=\lambda(s) \lambda(t) k(s \wedge t)-\int_{0}^{s \wedge t} \lambda^{2}(r) k^{\prime}(r) d r,
$$

for $s, t \in[0, T]$. Integrating by parts,

$$
\begin{aligned}
\tilde{U}_{s, t} & =\lambda(s) \lambda(t) k(s \wedge t)-\lambda^{2}(s \wedge t) k(s \wedge t)+2 \int_{0}^{s \wedge t} \lambda^{\prime}(r) \lambda(r) k(r) d r \\
& =2 \int_{0}^{s \wedge t} \lambda^{\prime}(r) \lambda(r) k(r) d r+\left(\lambda(s) \lambda(t)-\lambda^{2}(s \wedge t)\right) k(s \wedge t) .
\end{aligned}
$$

From this it is easy to see that

$$
\tilde{U}_{\bar{s} \bar{t}}=\int_{0}^{\bar{s}} d s \int_{0}^{\bar{t}} d t\left(\lambda^{\prime}(s) \lambda^{\prime}(t) k(s \wedge t)+\lambda(s \wedge t) \lambda^{\prime}(s \vee t) k^{\prime}(s \wedge t)\right)
$$

since for $\bar{s} \leq \bar{t}$ one has

$$
\begin{aligned}
& \int_{0}^{\bar{s}} d s \int_{0}^{\bar{t}} d t\left(\lambda^{\prime}(s) \lambda^{\prime}(t) k(s \wedge t)+\lambda(s \wedge t) \lambda^{\prime}(s \vee t) k^{\prime}(s \wedge t)\right) \\
= & \int_{0}^{\bar{s}}\left\{\int_{0}^{s}\left(\lambda^{\prime}(s) \lambda^{\prime}(t) k(t)+\lambda(t) \lambda^{\prime}(s) k^{\prime}(t)\right) d t\right. \\
& \left.+\int_{s}^{\bar{t}}\left(\lambda^{\prime}(s) \lambda^{\prime}(t) k(s)+\lambda(s) \lambda^{\prime}(t) k^{\prime}(s)\right) d t\right\} \\
= & \int_{0}^{\bar{s}} \lambda^{\prime}(s) \lambda(s) k(s) d s+\int_{0}^{\bar{s}}(\lambda(\bar{t})-\lambda(s)) \frac{d}{d s}(\lambda(s) k(s)) d s \\
= & 2 \int_{0}^{\bar{s}} \lambda^{\prime}(s) \lambda(s) k(s) d s+(\lambda(\bar{t})-\lambda(\bar{s})) \lambda(\bar{s}) k(\bar{s}) .
\end{aligned}
$$

This shows that $U \in \wedge^{2} \mathcal{H}^{1}$. Next note that

$$
\tilde{U}_{s, s}=2 \int_{0}^{s} \lambda^{\prime}(r) \lambda(r) k(r) d r
$$


by (38). By the definition, (28), of $Q$, (34), and using (36) again we see that for $0 \leq s \leq t \leq T$,

$$
\begin{aligned}
& {\left[\wedge^{2}\left(W^{-1}\right) Q(U)\right]_{s, t}:=\wedge^{2}\left(W_{s}^{-1}\right) W_{s}^{(2)} \int_{0}^{s}\left(W_{r}^{(2)}\right)^{-1} \mathcal{R}\left(U_{r r}\right) d r } \\
= & \wedge^{2}\left(W_{s}^{-1}\right) W_{s}^{(2)} \int_{0}^{s}\left(W_{r}^{(2)}\right)^{-1} \wedge^{2}\left(W_{r}\right) \frac{d}{d r} \tilde{U}_{r, r} d r \\
& -\wedge^{2}\left(W_{s}^{-1}\right) W_{s}^{(2)} \int_{0}^{s} \frac{d}{d r}\left[\left(W_{r}^{(2)}\right)^{-1} U_{r r}\right] d r \\
= & \wedge^{2}\left(W_{s}^{-1}\right) W_{s}^{(2)} \int_{0}^{s} 2\left(W_{r}^{(2)}\right)^{-1} \wedge^{2}\left(W_{r}\right) \lambda^{\prime}(r) \lambda(r) k(r) d r-\tilde{U}_{s, s} \\
= & \wedge^{2}\left(W_{s}^{-1}\right) W_{s}^{(2)} \int_{0}^{s} 2 \lambda^{\prime}(r) \lambda(r) V d r-\tilde{U}_{s, s} \\
= & \lambda^{2}(s) k(s)-2 \int_{0}^{s} \lambda^{\prime}(r) \lambda(r) k(r) d r \\
= & \int_{0}^{s}[\lambda(r)]^{2} k^{\prime}(r) d r .
\end{aligned}
$$

Finally add (37) to the above to obtain the required identity (35).

To obtain (31) we can assume $V$ to be primitive, $V=b^{1} \wedge b^{2}$ say. By (18) the divergence of $Z$ is just the conditional expectation $\bar{J}$ of $J$ for $J=T \mathcal{I}\left(\operatorname{div}\left(h^{1} \wedge\right.\right.$ $\left.h^{2}\right)$ ) where $\dot{h}_{t}^{j}=\dot{\lambda}(t) Y_{x_{t}} T \xi_{t}\left(b^{j}\right)$. By Theorem 2.1 and Proposition 3.1

$$
\begin{aligned}
J_{t} & =T \mathcal{I}_{t}\left(-\int_{0}^{T}\left\langle\dot{h}_{r}^{1}, d B_{r}\right\rangle h^{2}+\int_{0}^{T}\left\langle\dot{h}_{r}^{2}, d B_{r}\right\rangle h^{1}\right) \\
& =J_{t}^{0}+J_{t}^{1}
\end{aligned}
$$

where

$$
\begin{aligned}
J_{t}^{0}= & T \mathcal{I}_{t}\left(-\int_{t}^{T}\left\langle\dot{h}_{r}^{1}, d B_{r}\right\rangle h_{\cdot}^{2}+\int_{t}^{T}\left\langle\dot{h}_{r}^{2}, d B_{r}\right\rangle h_{\cdot}^{1}\right) \\
= & -\int_{t}^{T}\left\langle\dot{\lambda}(r) T \xi_{r}^{t}\left(T \xi_{t}\left(b^{1}\right)\right), d x_{r}\right\rangle \lambda(t) T \xi_{t}\left(b^{2}\right) \\
& +\int_{t}^{T}\left\langle\dot{\lambda}(r) T \xi_{r}^{t}\left(T \xi_{t}\left(b^{2}\right)\right), d x_{r}\right\rangle \lambda(t) T \xi_{t}\left(b^{1}\right) .
\end{aligned}
$$

Therefore

$$
\mathbb{E}\left\{J_{t}^{0} \mid \mathcal{F}^{x_{0}} \vee \mathcal{F}_{t}\right\}=-\lambda(t) \int_{t}^{T} \dot{\lambda}(r) \iota_{\left\langle W_{r}^{t-}, d x_{r}\right\rangle} \wedge^{2}\left(T \xi_{t}\right)\left(b^{1} \wedge b^{2}\right)
$$


giving

$$
\bar{J}_{t}^{0}=\int_{t}^{T}\left(\mathbf{1} \otimes\left\langle\frac{\mathbb{D}}{\partial r}-, d x_{r}\right\rangle\right) Z_{t, r}
$$

by (32).

The second term $J_{t}^{1}$ could be treated as in Corollary 3.5 below, but to get the form (31) observe

$$
J_{t}^{1}=-\int_{0}^{t} \lambda(t) \dot{\lambda}(r)\left(\left\langle-, d x_{r}\right\rangle \otimes \mathbf{1}\right)\left(\wedge^{2}(T \xi)\left(b^{1} \wedge b^{2}\right)\right)_{r t}
$$

and so by (27)

$$
\left.\bar{J}_{t}^{1}=-\int_{0}^{t} \lambda(t) \dot{\lambda}(r)\left(\left\langle-, d x_{r}\right\rangle \otimes \mathbf{1}\right)\left(\mathbf{1} \otimes W_{t}^{r}\right) W_{r}^{(2)}\left(b^{1} \wedge b^{2}\right)\right)_{r t} .
$$

Here we can interpret the, apparently adapted, integral by using $W_{t}^{r}=W_{t}\left(W_{r}\right)^{-1}$ and taking the term $\left(1 \otimes W_{t}\right)$ outside of the integral. Now

$$
\begin{aligned}
\left(\frac{\mathbb{D}}{\partial r} \otimes \mathbf{1}\right) Z_{r t}= & \frac{D}{\partial r}\left[\lambda(r) \lambda(t)\left(\mathbf{1} \otimes W_{t}^{r}\right) W_{r}^{(2)}\left(b^{1} \wedge b^{2}\right)\right] \\
& +\frac{1}{2}\left(\operatorname{Ric}^{\#} \otimes \mathbf{1}\right) Z_{r t}
\end{aligned}
$$

giving

$$
\begin{aligned}
& \dot{\lambda}(r) \lambda(t)\left(\mathbf{1} \otimes W_{t}^{r}\right) W_{r}^{(2)}\left(b^{1} \wedge b^{2}\right) \\
= & \left(\frac{\mathbb{D}}{\partial r} \otimes \mathbf{1}\right) Z_{r t}-\frac{1}{2}\left(R i c^{\#} \otimes \mathbf{1}\right) Z_{r t} \\
& -\lambda(r) \lambda(t)\left(1 \otimes W_{t}\right) \frac{D}{\partial r}\left(\mathbf{1} \otimes\left(W_{r}\right)^{-1}\right) W_{r}^{(2)}\left(b^{1} \wedge b^{2}\right),
\end{aligned}
$$

but

$$
\begin{aligned}
& \frac{D}{\partial r}\left(\mathbf{1} \otimes\left(W_{r}\right)^{-1}\right) W_{r}^{(2)}\left(b^{1} \wedge b^{2}\right) \\
& =\left(W_{r} \otimes \mathbf{1}\right) \frac{D}{\partial r}\left(\wedge^{2} W_{r}^{-1}\right) W_{r}^{(2)}\left(b^{1} \wedge b^{2}\right)-\frac{1}{2}\left(R i c^{\#} \otimes \mathbf{1}\right)\left(\mathbf{1} \otimes W_{r}^{-1}\right) W_{r}^{(2)}\left(b^{1} \wedge b^{2}\right) \\
& =\left(\mathbf{1} \otimes W_{r}^{-1}\right) \mathcal{R} W_{r}^{(2)}\left(b^{1} \wedge b^{2}\right)-\frac{1}{2}\left(R i c^{\#} \otimes \mathbf{1}\right)\left(\mathbf{1} \otimes W_{r}^{-1}\right) W_{r}^{(2)}\left(b^{1} \wedge b^{2}\right)
\end{aligned}
$$

by (36), and so

$$
\dot{\lambda}(r) \lambda(t)\left(\mathbf{1} \otimes W_{t}^{r}\right) W_{r}^{(2)}\left(b^{1} \wedge b^{2}\right)=\left(\frac{\mathbb{D}}{\partial r} \otimes \mathbf{1}\right) Z_{r t}-\left(\mathbf{1} \otimes W_{t}^{r}\right) \mathcal{R}\left(\mathbf{1} \otimes W_{t}^{r}\right)^{-1} Z_{r t} .
$$


The result follows noting that $\left(1 \otimes W_{t}^{r}\right)^{-1} Z_{r t}$ is $\mathcal{F}_{r}^{x_{0}}$-measurable and the factor $\left(\mathbf{1} \otimes W_{t}\right)$ in $\left(\mathbf{1} \otimes W_{t}^{r}\right)=\left(\mathbf{1} \otimes W_{t}\right)\left(\mathbf{1} \otimes\left(W_{r}\right)^{-1}\right)$ can be taken out of the stochastic integrals to make them Itô integrals.

\section{Remark 3.3.}

(1). The formula (30) in Proposition 3.3 demonstrates the alternative characterisation of $\mathcal{H}^{2}$ from [EL] which states that $V$ is in $\mathcal{H}^{2}$ if and only if $V-\mathbb{R}(V)$ is in $\wedge^{2} \mathcal{H}^{1}$ where $\mathbb{R}$ is the curvature operator of the damped Markovian connection on $\mathcal{H}^{1}$.

(2). Note that for the proof of (30) we could allow $V: C_{x_{0}} M \rightarrow \wedge^{2} T_{x_{0}} M$ to be nonconstant provided it is in $L^{1+\epsilon}$ for some $\epsilon>0$. Our filtration in calculating (27) can be chosen to be $\left\{\mathcal{F}_{t} \vee \mathcal{F}^{x_{0}}: 0 \leq t \leq T\right\}$ as in [EL96]. Now let $V:[0, T] \times[0, T] \rightarrow \wedge^{2} T_{x_{0}} M$ be such that $V_{s, t}=\int_{0}^{s} \int_{0}^{t} Z_{a, b} d a d b$ some $Z \in$ $L^{2}\left([0, T] \times[0, T] \times C_{x_{0}} M \rightarrow \wedge^{2} T_{x_{0}} M\right)$ symmetric in $(s, t) \in[0, T] \times[0, T]$. As a corollary we see, for $0 \leq s \leq t \leq T$,

$$
\mathbb{E}\left\{\wedge^{2}(T \xi)(V) \mid x_{s}: 0 \leq s \leq t \leq T\right\}=U+Q(U)
$$

for

$$
U_{s, t}=\left(\mathbf{1} \otimes W_{t}^{s}\right) W_{s}^{(2)}\left(V_{s, t}\right)-\left(W_{s} \otimes W_{t}\right) \int_{0}^{s} \wedge^{2}\left(W_{r}\right)^{-1} \mathcal{R}\left(W_{r}^{(2)}\left(V_{r r}\right)\right) d r .
$$

Moreover $U$ is a section of $\wedge^{2} \mathcal{H}^{1}$ and so $U+Q(U)$ is a section of $\mathcal{H}^{2}$. This follows from the proposition by polarization for $V_{s, t}=\left(\lambda^{1}(s) \lambda^{2}(t)+\lambda^{2}(s) \lambda^{1}(t)\right) b$, some $b \in L^{2}\left(C_{x_{0}} M ; \wedge^{2} T_{x_{0}} M\right)$ and then for general $V$ by continuity since we can consider such $V$ as elements of the completed tensor product $\left[\odot^{2} L_{0}^{2,1}([0, T] ; \mathbb{R})\right] \otimes$ $L^{2}\left(C_{x_{0}} M ; \wedge^{2} T_{x_{0}} M\right)$, where $\odot$ refers to the symmetric tensor product. Note the mapping

$$
\begin{aligned}
& \odot^{2} L_{0}^{2,1}([0, T] ; \mathbb{R}) \otimes L^{2}\left(C_{x_{0}} M ; \wedge^{2} T_{x_{0}} M\right) \\
& \stackrel{\Theta}{\longrightarrow} L^{2}\left(C_{x_{0}} M ; \wedge^{2}\left(L_{0}^{2,1}(0, T] ; \mathbb{R}\right) \otimes T_{x_{0}} M\right) \\
& \simeq L^{2}\left(C_{x_{0}} M ; \wedge^{2}\left(L_{0}^{2,1}\left(T_{x_{0}} M\right)\right)\right.
\end{aligned}
$$

given by

$$
\begin{aligned}
& \Theta((u \odot v) \otimes f(\alpha \wedge \beta))(\sigma) \\
& =\frac{1}{2} f(\sigma)[(u \otimes \alpha) \wedge(v \otimes \beta)+(v \otimes \alpha) \wedge(u \otimes \beta)], \sigma \in C_{x_{0}} M
\end{aligned}
$$


where $f(\cdot)(\alpha \wedge \beta) \in L^{2}\left(C_{x_{0}} M ; \wedge^{2} T_{x_{0}} M\right) \simeq L^{2}\left(C_{x_{0}} M ; \mathbb{R}\right) \otimes \wedge^{2} T_{x_{0}} M$, $\alpha, \beta \in T_{x_{0}} M, f \in L^{2}\left(C_{x_{0}} M ; \mathbb{R}\right)$.

In this generality the stochastic integrals in (31) will need to be treated more carefully and if $V$ is non-random an additional term involving its $\mathrm{H}$-derivative will be involved in the divergence.

3.3 From Integration by parts to Bismut type formulae. First we shall give an extension of the non-intrinsic formula (4). For this consider a general nondegenerate stochastic differential equation (5) with smooth coefficients. For $\left\{\xi_{t}\right.$ : $0 \leq t<\infty\}$, its flow of diffeomorphisms of the manifold, consider the semigroup described in $\S 1$, given by

$$
P_{t} \phi=\mathbb{E} \xi_{t}^{*}(\phi) .
$$

Since $M$ is compact $d\left(P_{t} \phi\right)=P_{t}(d \phi)$ when $\phi$ is $C^{1}$. The generator $\mathcal{A}$ of $\left\{P_{t}\right\}_{t \geq 0}$ is given on smooth forms by (11) above.

Proposition 3.4 For any bounded measurable form $\phi$ on $M$ and $L^{1}$ function $\rho$ : $[0, t] \rightarrow \mathbb{R}$ with $\int_{0}^{t} \rho(r) d r \neq 0$,

$$
\begin{aligned}
& d\left(P_{t} \phi\right)\left(b^{1} \wedge \cdots \wedge b^{q+1}\right) \\
& =\left(\int_{0}^{t} \rho(r) d r\right)^{-1} \mathbb{E} \sum_{j=1}^{q+1}(-1)^{j+1} \int_{0}^{t} \rho(s)\left\langle T \xi_{s}\left(b^{j}\right), X\left(x_{s}\right) d B_{s}\right\rangle_{x_{s}} \cdot \\
& -\left(\int_{0}^{t} \rho(r) d r\right)^{-1} \sum_{1 \leq i<j \leq q+1}(-1)^{i+j+1} \mathbb{E} \xi_{t}^{*}(\phi) \\
& \left(\int_{0}^{t} \rho(s) T \xi_{s}^{-1}\left(\breve{T}\left(T \xi_{s}\left(b^{i}\right), T \xi_{s}\left(b^{j}\right)\right)\right) d s \wedge b^{1} \wedge \cdots \wedge \widehat{b^{j}} \wedge \wedge \wedge b^{q+1}\right)
\end{aligned}
$$

where $\breve{T}: T M \oplus T M \rightarrow T M$ is the torsion of the connection $\breve{\nabla}$ given by (8).

Proof. Define $h^{i}, i=1$ to $q+1$, by (19). Set $h=h^{1} \wedge \cdots \wedge h^{q+1}$ and $b=b^{1} \wedge \cdots \wedge$ $b^{q+1}$. Arguing as in Theorem 2.2, but without taking conditional expectations we 
see, for $\phi$ a $C^{1} q$-form,

$$
\begin{aligned}
d\left(P_{t} \phi\right)(b) & =P_{t}(d \phi)(b) \\
& =\mathbb{E} \xi_{t}^{*}(d \phi)(b)=\left(\int_{0}^{T} \rho(r) d r\right)^{-q-1} \mathbb{E} d \phi\left(\wedge^{q+1}(T \mathcal{I})(h)_{t, \ldots, t}\right) \\
& =-\left(\int_{0}^{T} \rho(r) d r\right)^{-q-1} \mathbb{E} \phi\left(\wedge^{q}(T \mathcal{I})(\operatorname{divh})_{t, \ldots, t}\right) .
\end{aligned}
$$

By Shigekawa's result, Theorem 2.1, formula (41) follows using Proposition 3.1, equation (16), (26), and the fact that $\int_{0}^{t}\left\langle\dot{h}_{s}^{i}, d B_{s}\right\rangle=\int_{0}^{t} \rho(s)\left\langle T \xi_{s}\left(b^{i}\right), X\left(x_{s}\right) d B_{s}\right\rangle_{x_{s}}$. By continuity it also holds for bounded measurable $\phi$.

To obtain an intrinsic formula from (41), we shall take conditional expectations, which can easily be done if the torsion $\breve{T}=X(d Y)$ is invariant under the flow (e.g. for certain homogeneous spaces). In this case formula (41) becomes:

$$
\begin{aligned}
& d\left(P_{t} \phi\right)\left(b^{1} \wedge \cdots \wedge b^{q+1}\right) \\
& =\left(\int_{0}^{t} \rho(r) d r\right)^{-1} \mathbb{E} \sum_{j=1}^{q+1}(-1)^{j+1} \int_{0}^{t} \rho(s)\left\langle T \xi_{s}\left(b^{j}\right), X\left(x_{s}\right) d B_{s}\right\rangle_{x_{s}} \cdot \\
& \xi_{t}^{*}(\phi)\left(b^{1} \wedge \cdots \wedge \widehat{b^{j}} \wedge \cdots \wedge b^{q+1}\right) \\
& \left.-\sum_{1 \leq i<j \leq q+1}(-1)^{i+j+1} \mathbb{E}\left(\xi_{t}^{*}(\phi)\left(\breve{T}\left(b^{i}, b^{j}\right) \wedge b^{1} \wedge \cdots \wedge \widehat{b^{i}} \cdots \wedge \widehat{b^{j}} \cdots \wedge b^{q+1}\right)\right)\right) .
\end{aligned}
$$

Let $\breve{W}_{t}^{A, q}: \wedge^{q} T_{x_{0}} M \rightarrow \wedge^{q} T_{x_{t}} M$, the damped parallel translation of $q$ vectors, defined by:

$$
\left\{\begin{aligned}
\frac{\hat{D}}{\partial s}\left(\breve{W}_{s}^{A, q}\left(V_{0}\right)\right) & =-\frac{1}{2} \breve{\mathcal{R}}_{x_{0}}^{q}\left(\breve{W}_{s}^{A, q}\left(V_{0}\right)\right)+d \wedge^{q}(\breve{\nabla} A)\left(\breve{W}_{s}^{A, q}\left(V_{0}\right)\right) \\
\breve{W}_{0}^{A, q}\left(V_{0}\right) & =V_{0} .
\end{aligned}\right.
$$

Here $\frac{\hat{D}}{\partial s}$ refers to covariant differentiation using the connection $\hat{\nabla}$, the adjoint connection of $\breve{\nabla}$. Since the conditional expectation of $\wedge^{q} T \xi_{t}$ is given by

$$
\overline{\wedge^{q} T \xi_{t}}(-)=\breve{W}_{t}^{A, q}(-)
$$


by Theorem 3.3.7 in [ELL99], or Theorem A in [EY93] for the Levi-Civita connection, we only need to worry about the first term on the right hand side of equation (42). Set

$U_{t}=\int_{0}^{t} \rho(s) \sum_{j=1}^{q+1}(-1)^{j+1}\left\langle T \xi_{s}\left(b^{j}\right), X\left(x_{s}\right) d B_{s}\right\rangle \cdot \wedge^{q} T \xi_{t}\left(b^{1} \wedge \cdots \wedge \widehat{b^{j}} \wedge \cdots \wedge b^{q+1}\right)$

and

$$
V_{t}^{j}=\wedge^{q} T \xi_{t}\left(b^{1} \wedge \cdots \wedge \widehat{b^{j}} \wedge \cdots \wedge b^{q+1}\right) .
$$

Then, e.g. by (3.3.10) in [ELL99],

$$
\begin{aligned}
\hat{D} U_{t}= & \sum_{j=1}^{q+1}(-1)^{j+1} \rho(t)\left\langle T \xi_{t}\left(b^{j}\right), X\left(x_{t}\right) d B_{t}\right\rangle \cdot V_{t}^{j} \\
& \left.+\sum_{j=1}^{q+1}(-1)^{j+1} \int_{0}^{t} \rho(s)\left\langle T \xi_{s}\left(b^{j}\right), X\left(x_{s}\right) d B_{s}\right\rangle \cdot d \wedge^{q}\left(\breve{\nabla} X(-) d B_{t}\right)\left(V_{t}^{j}\right)\right) \\
& +\sum_{j=1}^{q+1}(-1)^{j+1} \int_{0}^{t} \rho(s)\left\langle T \xi_{s}\left(b^{j}\right), X\left(x_{s}\right) d B_{s}\right\rangle \cdot \\
= & \sum_{j=1}^{q+1}(-1)^{j+1} \rho(t)\left\langle T \xi_{t}\left(b^{j}\right), X\left(x_{t}\right) d B_{t}\right\rangle \cdot V_{t}^{j} \\
& \left.\left.+d \wedge^{q}\left(\breve{\nabla} X(-) d B_{t}\right)\left(U_{t}\right)\right)-\frac{1}{2}\left(\breve{R}^{q}\right)^{*}\left(U_{t}\right) d t+d \wedge^{q} \breve{\nabla} A\left(V_{t}^{j}\right) d t\right) d t,
\end{aligned}
$$

Taking the conditional expectation of the above equation, as in the proof of Proposition 3.3.7 in [ELL99] or that of Theorem A in [EY93], we have 


$$
\begin{aligned}
\hat{D} \overline{U_{t}}= & \sum_{j=1}^{q+1}(-1)^{j+1} \rho(t)\left\langle T \xi_{t}\left(b^{j}\right), X\left(x_{t}\right) d B_{t}\right\rangle \cdot \wedge^{q} T \xi_{t}\left(b^{1} \wedge \cdots \wedge \widehat{b^{j}} \wedge \cdots \wedge b^{q+1}\right) \\
& -\frac{1}{2}\left(\breve{R}^{q}\right)^{*}\left(\overline{U_{t}}\right) d t+d \wedge^{q} \breve{\nabla} A\left(\overline{U_{t}}\right) d t \\
= & \rho(t) \iota_{\left\langle-, X\left(x_{t}\right) d B_{t}\right\rangle} \overline{\wedge^{q+1} T \xi_{t}\left(b^{1} \wedge \cdots \wedge b^{q+1}\right)}-\frac{1}{2}\left(\breve{R}^{q}\right)^{*}\left(\overline{U_{t}}\right) d t+d \wedge^{q} \breve{\nabla} A\left(\overline{U_{t}}\right) d t \\
= & \rho(t) \iota_{\left\langle-, X\left(x_{t}\right) d B_{t}\right\rangle} \breve{W}_{t}^{A, q+1}\left(b^{1} \wedge \cdots \wedge b^{q+1}\right)-\frac{1}{2}\left(\breve{R}^{q}\right)^{*}\left(\overline{U_{t}}\right) d t+d \wedge^{q} \breve{\nabla} A\left(\overline{U_{t}}\right) d t \\
= & \rho(t) \iota_{\left\langle-, \breve{/} t d \breve{B}_{t}\right\rangle} \breve{W}_{t}^{A, q+1}\left(b^{1} \wedge \cdots \wedge b^{q+1}\right)-\frac{1}{2}\left(\breve{R}^{q}\right)^{*}\left(\overline{U_{t}}\right) d t+d \wedge^{q} \breve{\nabla} A\left(\overline{U_{t}}\right) d t
\end{aligned}
$$

Here $/ /{ }_{t}$ denotes parallel translation corresponding to the connection $\breve{\nabla}$ and $\left(\breve{B}_{s}\right)$ is the stochastic anti-development Brownian motion on $T_{x_{0}} M$, i.e. the martingale part of $\int_{0} \breve{/}_{s}^{-1} \circ d x_{s}$. Solve the equation to obtain

$$
\overline{U_{t}}=\breve{W}_{t}^{A, q} \int_{0}^{t}\left(\breve{W}_{s}^{A, q}\right)^{-1} \rho(s) \iota_{\left\langle-, \breve{/}_{s} d \breve{B}_{s}\right\rangle} \breve{W}_{s}^{A, q+1}\left(b^{1} \wedge \cdots \wedge b^{q+1}\right) d s .
$$

Finally we arrive at:

Corollary 3.5 Suppose that the torsion $\breve{T} \equiv X d Y$ is invariant under the flow $\xi_{t}$. Then for $b^{i} \in T_{x_{0}} M, i=1, \ldots, q+1$,

$$
\begin{aligned}
& d\left(P_{t} \phi\right)\left(b^{1} \wedge \cdots \wedge b^{q+1}\right) \\
& =\left(\int_{0}^{t} \rho(r) d r\right)^{-1} \mathbb{E} \phi\left(\breve{W}_{t}^{A, q} \int_{0}^{t} \rho(s)\left(\breve{W}_{s}^{A, q}\right)^{-1} \iota_{\left\langle-, \breve{l}_{s} d \breve{B}_{s}\right\rangle} \breve{W}_{s}^{A, q+1}\left(b^{1} \wedge \cdots \wedge b^{q+1}\right)\right) \\
& -\phi\left(\breve{W}_{t}^{A, q}\left(\sum_{1 \leq i<j \leq q+1}(-1)^{i+j+1} \breve{T}\left(b^{i}, b^{j}\right) \wedge b^{1} \wedge \cdots \wedge \widehat{b}^{i} \cdots \wedge \widehat{b}^{j} \cdots \wedge b^{q+1}\right)\right) .
\end{aligned}
$$

Note that in the non-invariant case, an analogous proof to that of Corollary 3.5 leads to the intrinsic formula below. If $V$ is a $q+1$ vector, we define $\iota_{\breve{T}} V$ to be the operator from $\wedge^{q+1} T M \rightarrow \wedge^{q} T M$ which restricted to primitive vectors is given by:

$$
\iota_{\breve{T}}\left(b^{1} \wedge \cdots \wedge b^{q+1}\right)=\sum_{1 \leq i<j \leq q+1}(-1)^{i+j+1} \breve{T}\left(b^{i}, b^{j}\right) \wedge b^{1} \wedge \cdots \wedge \widehat{b^{i}} \cdots \wedge \widehat{b^{j}} \cdots \wedge b^{q+1} .
$$


Corollary 3.6 Let $b$ be a $q$ vector in $\wedge^{q+1} T_{x_{0}} M$, then

$$
\begin{aligned}
& \left(\int_{0}^{t} \rho(r) d r\right) d\left(P_{t} \phi\right)(b) \\
= & \mathbb{E} \phi\left(\breve{W}_{t}^{A, q} \int_{0}^{t} \rho(s)\left(\breve{W}_{s}^{A, q}\right)^{-1}\left(\iota_{\left\langle-, \breve{l}_{s} d \breve{B}_{s}\right\rangle} W_{s}^{A, q+1}(b)+\iota_{\breve{T}} \breve{W}_{s}^{A, q+1}(b) d s\right)\right) .
\end{aligned}
$$

Proof. We only need to worry about the last term of (41), since the previous term is as in Corollary 3.5. For this

$$
\begin{aligned}
& \sum_{1 \leq i<j \leq q+1}(-1)^{i+j+1} \\
& \mathbb{E}\left(\xi_{t}^{*}\right)(\phi)\left(\int_{0}^{t} \rho(s) T \xi_{s}^{-1}\left(\breve{T}\left(T \xi_{s}\left(b^{i}\right), T \xi_{s}\left(b^{j}\right)\right)\right) d s \wedge b^{1} \wedge \cdots \wedge \widehat{b^{i}} \cdots \wedge \widehat{b^{j}} \cdots \wedge b^{q+1}\right) \\
& =\mathbb{E} \phi\left(\sum_{1 \leq i<j \leq q+1}(-1)^{i+j+1} T \xi_{t} \int_{0}^{t} \rho(s) T \xi_{s}^{-1}\left(\breve{T}\left(T \xi_{s}\left(b^{i}\right), T \xi_{s}\left(b^{j}\right)\right)\right) d s \wedge\right. \\
& \left.\left.T \xi_{t}\left(b^{1}\right) \wedge \cdots \wedge \widehat{T \xi_{t}\left(b^{i}\right)} \cdots \wedge \widehat{T \xi_{t}\left(b^{j}\right.}\right) \cdots \wedge T \xi_{t}\left(b^{q+1}\right)\right) .
\end{aligned}
$$

Set

$$
\begin{aligned}
U_{t}= & \sum_{1 \leq i<j \leq q+1}(-1)^{i+j+1} T \xi_{t} \int_{0}^{t} \rho(s) T \xi_{s}^{-1}\left(\breve{T}\left(T \xi_{s}\left(b^{i}\right), T \xi_{s}\left(b^{j}\right)\right)\right) d s \wedge \\
& \left.T \xi_{t}\left(b^{1}\right) \wedge \cdots \wedge \widehat{T \xi_{t}\left(b^{i}\right)} \cdots \wedge \widehat{T \xi_{t}\left(b^{j}\right.}\right) \cdots \wedge T \xi_{t}\left(b^{q+1}\right) .
\end{aligned}
$$

Then after covariant differentiation and filtering we have

$$
\begin{aligned}
\hat{D} \overline{U_{t}}= & \sum_{1 \leq i<j \leq q+1}(-1)^{i+j+1} \rho(t) \cdot \\
& \bar{T}\left(T \xi_{t}\left(b^{i}\right), T \xi_{t}\left(b^{j}\right)\right) \wedge T \xi_{t}\left(b^{1}\right) \wedge \cdots \wedge \widehat{T \xi_{t}\left(b^{i}\right)} \cdots \wedge \widehat{T \xi_{t}\left(b^{j}\right)} \cdots \wedge T \xi_{t}\left(b^{q+1}\right) \\
& -\frac{1}{2}\left(\breve{R}^{q}\right)^{*}\left(\overline{U_{t}}\right) d t+d \wedge^{q} \breve{\nabla} A\left(\overline{U_{t}}\right) d t \\
= & \rho(t) \overline{\iota_{\breve{T}} \wedge^{q+1} T \xi_{t}\left(b^{1} \wedge \cdots \wedge b^{q+1}\right)}-\frac{1}{2}\left(\breve{R}^{q}\right)^{*}\left(\overline{U_{t}}\right) d t+d \wedge^{q} \breve{\nabla} A\left(\overline{U_{t}}\right) d t \\
= & \rho(t) \iota_{\breve{T}} \breve{W}_{t}^{A, q+1}(b)-\frac{1}{2}\left(\breve{R}^{q}\right)^{*}\left(\overline{U_{t}}\right) d t+d \wedge^{q} \breve{\nabla} A\left(\overline{U_{t}}\right) d t,
\end{aligned}
$$


giving

$$
\bar{U}_{t}=\breve{W}_{t}^{A, q} \int_{0}^{t} \rho(s)\left(\breve{W}_{s}^{A, q}\right)^{-1} \iota_{\breve{T}} \breve{W}_{s}^{A, q+1}(b) d s .
$$

The required equation now follows.

\section{Special cases}

(1) When the connection $\breve{\nabla}$ defined by (7) is the Levi-Civita connection and $\rho(t) \equiv 1$, formula (41) essentially reduces to (4), but in this case $P_{t}$ has generator given by $\frac{1}{2} \Delta+\mathcal{L}_{A}$ on smooth forms.

(2) For a left invariant stochastic differential equation on a Lie group $G$ with bi-invariant metric and $A \equiv 0$, formula (41) reduces to

$$
\begin{aligned}
& d\left(P_{t} \phi\right)\left(b^{1} \wedge \cdots \wedge b^{q+1}\right) \\
& =\left(\int_{0}^{t} \rho(r) d r\right)^{-1} \mathbb{E}\left(\left\langle\int_{0}^{t} a d\left(x_{s}\right) d B_{s},-\right\rangle \wedge R_{x_{t}}^{*}(\phi)\right)\left(b^{1} \wedge \ldots \wedge b^{q+1}\right) \\
& -\sum_{1 \leq i<j \leq q+1}(-1)^{i+j} \mathbb{E} R_{x_{t}}^{*}(\phi)\left(\left[b^{i}, b^{j}\right] \wedge b^{1} \wedge \ldots \widehat{b^{i}} \wedge \ldots \widehat{b^{j}} \wedge \ldots b^{q+1}\right),
\end{aligned}
$$

for $x_{0}=e\left(\right.$ so $b^{j} \in \mathfrak{g}$, each $\left.j\right)$. In this case the generator of $P_{t}$ is $\frac{1}{2} \operatorname{trace} \nabla^{R} \nabla^{R}$ by (2.4.3) of [ELL99].

Formula (46) is intrinsic. It could have been deduced from the path space integration by parts formula of [FF97]. The $q=0$ case was given in [EL94].

(3) Another computable example comes when $A \equiv 0$ and $X$ is chosen so that $\breve{\nabla}$ has torsion $\breve{T}(u, v)=\frac{2}{n-1}(v \wedge u) Z(x)$ for $u, v \in T_{x} M$ and $Z$ a fixed vector field on $M$. Here $v \wedge u$ is the operator such that $(v \wedge u) Z(x)=\langle v, Z(x)\rangle_{x} u-$ $\langle u, Z(x)\rangle_{x} v$. This connection was used by [IW81]. In [ELL99], example 2.3.5 (though there is a minor misprint in the formula written there), the generator on $q$-forms is shown to be $\frac{1}{2} \Delta+\mathcal{L}_{\left[\frac{2(q-1)}{n-1}-1\right] Z}-\frac{2}{n-1} \iota_{Z} d$. Set $Z^{\#}=$ $\langle Z(x),-\rangle_{x}$. The term involving the torsion in (41) reduces to

$$
\begin{aligned}
& \left(\int_{0}^{t} \rho(r) d r\right)^{-1} \frac{4}{n-1} \mathbb{E} \xi_{t}^{*}\left(Z^{\#} \wedge \phi\right)\left(b^{1} \wedge \cdots \wedge b^{q+1}\right) \\
& =\frac{4}{(n-1) \int_{0}^{t} \rho(r) d r} P_{t}\left(Z^{\#} \wedge \phi\right) .
\end{aligned}
$$


In this case we have:

$$
\begin{aligned}
& d\left(P_{t} \phi\right)\left(b^{1} \wedge \cdots \wedge b^{q+1}\right) \\
& =\left(\int_{0}^{t} \rho(r) d r\right)^{-1} \mathbb{E} \phi\left(\int_{0}^{t} \rho(s) \iota_{\left\langle-, \breve{l}_{s} d \breve{B}_{s}\right\rangle} W_{s}^{q+1}(b)\right) \\
& +\frac{4}{(n-1) \int_{0}^{t} \rho(r) d r}\left(Z^{\#} \wedge \phi\right)\left(b^{1} \wedge \cdots \wedge b^{q+1}\right) .
\end{aligned}
$$

\section{References}

[Air76] Hélène Airault. Subordination de processus dans le fibré tangent et formes harmoniques. C. R. Acad. Sci. Paris Sér. A-B, 282(22):Aiii, A1311-A1314, 1976.

[Bis81] J.-M. Bismut. Martingales, the Malliavin calculus and Hörmander's theorem. In Stochastic integrals (Proc. Sympos., Durham, 1980), Lecture Notes in Mathematics 851, pages 85-109. Springer, 1981.

[Bis84] J. M. Bismut. Large deviations and the Malliavin calculus. Progress in Math. 45. Birkhaúser, 1984.

[Dri92] B. K. Driver. A Cameron-Martin type quasi-invariance theorem for Brownian motion on a compact Riemannian manifold. J. Functional Analysis, 100:272-377, 1992.

[DT01] B. Driver and A. Thalmaier. Heat equation derivative formulas for vector bundles. J. Funct. Anal., 183:42-108, 2001.

[EL] K. D. Elworthy and Xue-Mei Li. An $L^{2}$ theory for differential forms on path spaces. In preparation.

[EL94] K. D. Elworthy and Xue-Mei Li. Formulae for the derivatives of heat semigroups. J. Funct. Anal., 125(1):252-286, 1994.

[EL96] K. D. Elworthy and Xue-Mei Li. A class of integration by parts formulae in stochastic analysis I. In Itô's Stochastic Calculus and Probability Theory (dedicated to Itô on the occasion of his eightieth birthday). Springer, 1996. 
[EL98] K. David Elworthy and Xue-Mei Li. Bismut type formulae for differential forms. C. R. Acad. Sci., Sér/ I, Math. Paris, 327(1):87-92, 1998.

[EL00] K. D. Elworthy and Xue-Mei Li. Special Itô maps and an $L^{2}$ Hodge theory for one forms on path spaces. Canadian Mathematical Society Con. Proc., 28:145-162, 2000.

[ELJL97] K. D. Elworthy, Y. Le Jan, and Xue-Mei Li. Concerning the geometry of stochastic differential equations and stochastic flows. In K.D. Elworthy, S. Kusuoka, and I. Shigekawa, editors, New Trends in stochastic Analysis', Proc. Taniguchi Symposium, Sept. 1995, Charingworth. World Scientific Press, 1997.

[ELL99] K. D. Elworthy, Y. LeJan, and Xue-Mei Li. On the geometry of diffusion operators and stochastic flows, Lecture Notes in Mathematics 1720. Springer, 1999.

[Elw82] K. D. Elworthy. Stochastic Differential Equations on Manifolds, London Mathematical Society Lecture Notes Series 70. Cambridge University Press, 1982.

[Elw88] K. D. Elworthy. Geometric aspects of diffusions on manifolds. In P. L. Hennequin, editor, Ecole d'Eté de Probabilités de Saint-Flour XVXVII, 1985-1987. Lecture Notes in Mathematics 1362, volume 1362, pages 276-425. Springer-Verlag, 1988.

[Elw92] K. D. Elworthy. Stochastic flows on Riemannian manifolds. In M. A. Pinsky and V. Wihstutz, editors, Diffusion processes and related problems in analysis, volume II. Birkhauser Progress in Probability, pages 37-72. Birkhauser, Boston, 1992.

[EY93] K. D. Elworthy and M. Yor. Conditional expectations for derivatives of certain stochastic flows. In J. Azéma, P.A. Meyer, and M. Yor, editors, Sem. de Prob. XXVII. Lecture Notes in Mathematics 1557, pages 159172. Springer-Verlag, 1993.

[FF97] S. Z. Fang and J. Franchi. De Rham-Hodge-Kodaira operator on loop groups. J. Functional Analysis, 148:391-407, 1997. 
[IW81] N. Ikeda and S. Watanabe. Stochastic Differential Equations and Diffusion Processes. North-Holland, 1981.

[JL91] J. D. S. Jones and R. Léandre. $L^{p}$-Chen forms on loop spaces. In Stochastic analysis (Durham, 1990), pages 103-162. London Mathematical Society Lecture Notes Series 167. Cambridge University Press, Cambridge, 1991.

[Kus88] S. Kusuoka. Degree theorem in certain Wiener-Riemannian manifolds. In Stochastic Analysis: Japanese-French Seminar 1987. Lecture Notes in Mathematics, 1322, pages 93-108. Springer-Verlag, 1988.

[Kus92] S. Kusuoka. Analysis on Wiener spaces II, Differential forms. J. Funct. Anal., 103:229-274, 1992.

[Lan62] S. Lang. Introduction to differential manifolds. Interscience Publishers, 1962.

[Lea] R. Leandre. Analysis over loop space and topology. To appear in Mathematical Notes.

[Li92] Xue-Mei Li. Stochastic flows on noncompact manifolds. University of Warwick, 1992. Ph.D. thesis.

[Nor93] J. Norris. Path integral formulae for heat kernels and their derivatives. Probability Theory and Related Fields, 94:525-541, 1993.

[RS84] Diego Rapoport and Shlomo Sternberg. On the interaction of spin and torsion. Ann. Physics, 158(2), 1984.

[Shi86] I. Shigekawa. De Rham-Hodge-Kodaira's decomposition on an abstract Wiener space. J. Math. Kyoto Univ., 26(2):191-202, 1986.

K. D. ELWORTHY, MATHEMATICS INSTITUTE, WARWICK UNIVERSITY, COVENTRY CV4 7AL, UK

XUE-MEI LI, DEPARTMENT OF COMPUTING AND MATHEMATICS, THE NOTTINGHAM TRENT UNIVERSITY, BURTON STREET, NOTTINGHAM NG1 4BU, U.K. and DEPARTMENT OF MATHEMATICS, UNIVERSITY OF CONNECTICUT, 196 AUDITORIUM ROAD, STORRS, CT 06269, USA. email address: xmli@math.uconn.edu 